\title{
Synthesis of Dihydrothiophene, Thiophene, and Their Selenium Analogues Carrying Four Phosphoryl Groups
}

\author{
Shigeru Sasaki, ${ }^{*}$ Kazutaka Adachi and Masaaki Yoshifuji \\ Department of Chemistry, Graduate School of Science, Tohoku \\ University, Aoba, Sendai 980-8578, Japan \\ sasaki@mail.tains.tohoku.ac.jp
}

\section{Experimental}

\section{General methods}

${ }^{1} \mathrm{H},{ }^{13} \mathrm{C},{ }^{31} \mathrm{P}$, and ${ }^{77} \mathrm{Se}$ NMR spectra were measured on a Bruker AV400 spectrometer. ${ }^{1} \mathrm{H}$ and ${ }^{13} \mathrm{C}$ NMR chemical shifts were calibrated by residual proton $(\delta 7.25 \mathrm{ppm}$ for $\mathrm{CDCl}_{3}, 3.30 \mathrm{ppm}$ for $\mathrm{CD}_{3} \mathrm{OD}$ ) and deuterated carbon ( $\delta 77.0 \mathrm{ppm}$ for $\mathrm{CDCl}_{3}, 49.0 \mathrm{ppm}$ for $\mathrm{CD}_{3} \mathrm{OD}$ ), respectively, and are expressed as $\delta$ downfield from external tetramethylsilane. ${ }^{31} \mathrm{P}$ and ${ }^{77} \mathrm{Se}$ NMR chemical shifts are expressed as $\delta$ downfield from external $85 \% \mathrm{H}_{3} \mathrm{PO}_{4}$ and dimethyl selenide, respectively. IR and UV-vis spectra were measured on a Horiba FT-300 and Shimadzu UV-3600, respectively. Mass spectra were measured on a Hitachi M-2500S with electron impact (EI) ionization at 70 $\mathrm{eV}$ or a JEOL HX-110 with fast atom bombardment (FAB) ionization using the $m$-nitrobenzyl alcohol matrix. FT-ICR-MS spectra were measured on a Bruker APEX3 with electron spray ionization (ESI). Microanalyses were performed at Research and Analytical Center for Giant Molecules, Graduate School of Science, Tohoku University. Merck silica gel 60 was used for column chromatography. All reactions were carried out under argon. Tetrahydrofuran and ether were distilled from sodium diphenylketyl under argon just prior to use. Bis(diethoxyphosphoryl)acetylene was prepared by the literature meshod. ${ }^{1}$ Sodium sulfide nonahydrate and sodium hydrosulfide hydrate were purchased from Wako Pure Chemical Industries and Ardrich, respectively. $m$ CPBA was purchased from Nacalai Tesque. 


\section{Synthesis}

\section{(Z)-1,2-Bis(diethoxyphosphoryl)-1-(methylthio)ethene (3).}<smiles>CCOC=C(S)COCC</smiles>

A mixture of 1 (314 mg, $1.06 \mathrm{mmol}$ ), sodium sulfide nonahydrate (765 mg, $1.02 \mathrm{mmol}$ ) in tetrahydrofuran $(5 \mathrm{~mL})$ was stirred for $12 \mathrm{~h}$ at $20^{\circ} \mathrm{C}$. Iodomethane $(0.07 \mathrm{~mL}, 1.07$ mmol) was added to the mixture at $20^{\circ} \mathrm{C}$ and the mixture was stirred for $10 \mathrm{~min}$. The mixture was submitted to column chromatography $\left(\mathrm{Al}_{2} \mathrm{O}_{3} /\right.$ ethyl acetate, acetone) to give crude 3 (7.1 mg, $0.289 \mathrm{mmol}, 2 \%)$. 3: yellow oil; ${ }^{1} \mathrm{H}$ NMR (400 $\mathrm{MHz}, \mathrm{CDCl}_{3}$, $293 \mathrm{~K}, \mathrm{ppm}) \delta 6.66\left(1 \mathrm{H}, \mathrm{dd}, J_{\mathrm{PH}}=24.3, J_{\mathrm{PH}}=15.5 \mathrm{~Hz},=\mathrm{CH}\right), 4.23-4.11\left(8 \mathrm{H}, \mathrm{m}, \mathrm{CH}_{2}\right)$, $2.57\left(3 \mathrm{H}, \mathrm{s}, \mathrm{SCH}_{3}\right), 1.39-1.30\left(12 \mathrm{H}, \mathrm{m}, \mathrm{CH}_{3}\right) ;{ }^{13} \mathrm{C} \mathrm{NMR}\left(101 \mathrm{MHz}, \mathrm{CDCl}_{3}, 293 \mathrm{~K}\right.$, ppm) $\delta 148.6(\mathrm{dd}, J=160.5,6.6 \mathrm{~Hz}$, alkene), 127.7 (dd, $J=177.5,14.7 \mathrm{~Hz}$, alkene), 63.6-62.7 (m, $\left.\underline{\mathrm{CH}}_{2}\right), 17.1\left(\mathrm{~s}, \mathrm{SCH}_{3}\right)$ 16.8-16.6 (m, $\left.\underline{\mathrm{CH}}_{3}\right) ;{ }^{31} \mathrm{P} \mathrm{NMR}\left(162 \mathrm{MHz}, \mathrm{CDCl}_{3}\right.$, $293 \mathrm{~K}, \mathrm{ppm}) \delta 13.7\left(\mathrm{~d},{ }^{3} J_{\mathrm{PP}}=85.5 \mathrm{~Hz}\right), 12.2\left(\mathrm{~d},{ }^{3} J_{\mathrm{PP}}=85.5 \mathrm{~Hz}\right) ; \mathrm{IR}(\mathrm{NaCl}) 1245$ $(v(\mathrm{PO})), 1024(v(\mathrm{POEt})) \mathrm{cm}^{-1}$; LRMS (rel. intensity, \%) $m / z 347\left(M^{+}+1,100\right), 301$ $\left(M^{+}-\right.$OEt, 87); FT-ICR-MS (ESI, positive) Found: $m / z$ 369.0660. Calcd for $\left[\mathrm{C}_{11} \mathrm{H}_{24} \mathrm{O}_{6} \mathrm{P}_{2} \mathrm{~S}+\mathrm{Na}\right]^{+}: 369.0661\left([M+\mathrm{Na}]^{+}\right)$.

\section{2,3,5-Tris(diethoxyphosphoryl)-trans-2,3-dihydrothiophene (4).}<smiles>CCOCCOCCOCCOCC</smiles>

A mixture of 1 (443 mg, $1.49 \mathrm{mmol})$, sodium sulfide nonahydrate (175 mg, 0.729 $\mathrm{mmol})$, in ether $(5 \mathrm{~mL})$ was stirred for $12 \mathrm{~h}$ at $20{ }^{\circ} \mathrm{C}$. The mixture was submitted to column chromatography $\left(\mathrm{SiO}_{2} /\right.$ ethyl acetate, acetone, ethanol) to give 4 (63.9 $\mathrm{mg}$, 0.129 mmol, 17\%). 4: yellow oil; ${ }^{1} \mathrm{H}$ NMR (400 $\left.\mathrm{MHz}, \mathrm{CDCl}_{3}, 293 \mathrm{~K}, \mathrm{ppm}\right) \delta 7.10$ $\left(1 \mathrm{H}, \mathrm{dd},{ }^{3} J_{\mathrm{PH}}=11.0 \mathrm{~Hz},{ }^{3} J_{\mathrm{PH}}=5.1 \mathrm{~Hz}, 4-\mathrm{CH}\right), 4.26\left(1 \mathrm{H}, \mathrm{ddm},{ }^{3} J_{\mathrm{PH}}=23.3 \mathrm{~Hz},{ }^{2} J_{\mathrm{PH}}=\right.$ $11.0 \mathrm{~Hz}, 2-\mathrm{CH}), 4.20-3.98\left(12 \mathrm{H}, \mathrm{m}, \mathrm{CH}_{2}\right), 3.82\left(1 \mathrm{H}, \mathrm{ddm},{ }^{3} J_{\mathrm{PH}}=21.2 \mathrm{~Hz},{ }^{2} J_{\mathrm{PH}}=21.2\right.$ $\mathrm{Hz}, 2-\mathrm{CH}), 1.32-1.22\left(18 \mathrm{H}, \mathrm{m}, \mathrm{CH}_{3}\right) ;{ }^{13} \mathrm{C} \mathrm{NMR}\left(101 \mathrm{MHz}, \mathrm{CDCl}_{3}, 293 \mathrm{~K}, \mathrm{ppm}\right) \delta$ $146.4\left(\mathrm{dd},{ }^{2} J_{\mathrm{PC}}=16.2 \mathrm{~Hz},{ }^{2} J_{\mathrm{PC}}=10.3 \mathrm{~Hz}, 4-\underline{\mathrm{C}}\right), 121.7\left(\mathrm{dd},{ }^{1} J_{\mathrm{PC}}=196.6 \mathrm{~Hz},{ }^{3} J_{\mathrm{PC}}=8.4\right.$ $\mathrm{Hz}, 5-\underline{\mathrm{C}}), 63.7\left(\mathrm{~d},{ }^{2} J_{\mathrm{PC}}=6.9 \mathrm{~Hz}, \underline{\mathrm{CH}}_{2}\right), 63.3\left(\mathrm{~d},{ }^{2} J_{\mathrm{PC}}=6.6 \mathrm{~Hz}, \underline{\mathrm{CH}}_{2}\right), 62.8\left(\mathrm{~d},{ }^{2} J_{\mathrm{PC}}=6.7\right.$ $\left.\mathrm{Hz}, \underline{\mathrm{CH}}_{2}\right), 62.7\left(\mathrm{~d},{ }^{2} J_{\mathrm{PC}}=6.5 \mathrm{~Hz}, \underline{\mathrm{CH}}_{2}\right), 62.1\left(\mathrm{~d},{ }^{2} J_{\mathrm{PC}}=6.1 \mathrm{~Hz}, \underline{\mathrm{CH}}_{2}\right), 62.0\left(\mathrm{~d},{ }^{2} J_{\mathrm{PC}}=5.5\right.$ $\left.\mathrm{Hz}, \underline{\mathrm{CH}}_{2}\right), 48.2\left(\mathrm{dd},{ }^{1} J_{\mathrm{PC}}=135.7 \mathrm{~Hz},{ }^{2} J_{\mathrm{PC}}=15.4 \mathrm{~Hz}, 2\right.$ or $\left.3-\underline{\mathrm{C}}\right), 45.3\left(\mathrm{dd},{ }^{1} J_{\mathrm{PC}}=143.8\right.$ $\mathrm{Hz},{ }^{2} J_{\mathrm{PC}}=11.4 \mathrm{~Hz}, 2$ or 3-$), 16.8-16.6\left(\mathrm{~m}, \underline{\mathrm{CH}_{3}}\right) ;{ }^{31} \mathrm{P} \mathrm{NMR}\left(162 \mathrm{MHz}, \mathrm{CDCl}_{3}, 293 \mathrm{~K}\right.$, 
ppm) $\delta 23.9\left(\mathrm{~d},{ }^{3} J_{\mathrm{PP}}=74.4 \mathrm{~Hz}, 2\right.$ or $\left.3-\mathrm{P}\right), 22.5\left(\mathrm{~d},{ }^{3} J_{\mathrm{PP}}=74.4 \mathrm{~Hz}, 2\right.$ or $\left.3-\mathrm{P}\right), 12.0(\mathrm{~s}$, 5-P); UV-Vis (methanol) $\lambda_{\max }(\varepsilon) 279$ (5600), 253sh (3500) nm; IR (NaCl) 1242 $(v(\mathrm{PO})), 1024(v(\mathrm{POEt})) \mathrm{cm}^{-1}$; FT-ICR-MS (ESI, positive) Found: $m / z 495.1134$. Calcd for $\mathrm{C}_{16} \mathrm{H}_{33} \mathrm{O}_{9} \mathrm{P}_{3} \mathrm{~S}^{+}$: $495.1131\left([\mathrm{M}]^{+}\right)$; Anal. Calcd for $\mathrm{C}_{16} \mathrm{H}_{33} \mathrm{O}_{9} \mathrm{P}_{3} \mathrm{~S} \cdot \mathrm{CHCl}_{3}$ : C, 33.27; H, 5.58; S, 5.22. Found: C, 33.47; H, 5.84; S, 5.17.

\section{2,3,4,5-Tetrakis(diethoxyphosphoryl)-trans-2,3-dihydrothiophene (5).}

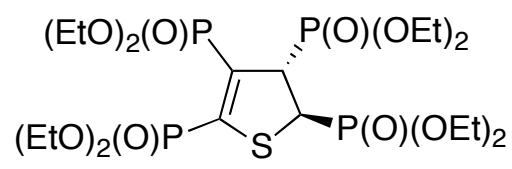

A mixture of 1 (1.87 g, $6.27 \mathrm{mmol})$, sodium hydrosulfide hydrate $(198 \mathrm{mg})$ in ether (5 $\mathrm{mL}$ ) was stirred for $12 \mathrm{~h}$ at $20{ }^{\circ} \mathrm{C}$. The mixture was concentrated under reduced pressure and purified by column chromatography $\left(\mathrm{SiO}_{2} /\right.$ ethyl acetate, acetone) to give 5 (1.01 g, $1.61 \mathrm{mmol}, 51 \%)$. 5: colorless oil; ${ }^{1} \mathrm{H} \mathrm{NMR}\left(400 \mathrm{MHz}, \mathrm{CDCl}_{3}, 293 \mathrm{~K}\right.$, ppm) $\delta 4.31-4.17\left(18 \mathrm{H}, \mathrm{m}, \mathrm{C} \underline{\mathrm{H}}+\underline{\mathrm{C}}_{2}\right), 1.36-1.16\left(24 \mathrm{H}, \mathrm{m}, \underline{\mathrm{C}}_{3}\right) ;{ }^{13} \mathrm{C} \mathrm{NMR}(101 \mathrm{MHz}$, $\left.\mathrm{CDCl}_{3}, 293 \mathrm{~K}, \mathrm{ppm}\right) \delta 148.5\left(\mathrm{ddd},{ }^{1} J_{\mathrm{PC}}=192.3 \mathrm{~Hz},{ }^{2} J_{\mathrm{PC}}=17.5 \mathrm{~Hz},{ }^{2} J_{\mathrm{PC}}=11.6 \mathrm{~Hz}, 4-\underline{\mathrm{C}}\right)$, $130.4\left(\mathrm{ddd},{ }^{1} J_{\mathrm{PC}}=192.3 \mathrm{~Hz},{ }^{2} J_{\mathrm{PC}}=13.9 \mathrm{~Hz},{ }^{2} J_{\mathrm{PC}}=10.2 \mathrm{~Hz}, 5-\underline{\mathrm{C}}\right), 63.9\left(\mathrm{~d},{ }^{2} J_{\mathrm{PC}}=7.0 \mathrm{~Hz}\right.$, $\left.\underline{\mathrm{CH}}_{2}\right), 63.7\left(\mathrm{~d},{ }^{2} J_{\mathrm{PC}}=6.0 \mathrm{~Hz}, \underline{\mathrm{CH}}_{2}\right), 63.5\left(\mathrm{~d},{ }^{2} J_{\mathrm{PC}}=6.5 \mathrm{~Hz}, \underline{\mathrm{CH}}_{2}\right), 63.4\left(\mathrm{~d},{ }^{2} J_{\mathrm{PC}}=5.8 \mathrm{~Hz}\right.$, $\left.\underline{\mathrm{CH}}_{2}\right), 63.2\left(\mathrm{~d},{ }^{2} J_{\mathrm{PC}}=6.5 \mathrm{~Hz}, \underline{\mathrm{CH}}_{2}\right), 63.1\left(\mathrm{~d},{ }^{2} J_{\mathrm{PC}}=6.5 \mathrm{~Hz}, \underline{\mathrm{CH}}_{2}\right), 62.73\left(\mathrm{~d},{ }^{2} J_{\mathrm{PC}}=6.3 \mathrm{~Hz}\right.$, $\left.\underline{\mathrm{CH}}_{2}\right), 62.66\left(\mathrm{~d},{ }^{2} J_{\mathrm{PC}}=7.1 \mathrm{~Hz}, \underline{\mathrm{CH}}_{2}\right), 53.9\left(\mathrm{dddd},{ }^{1} J_{\mathrm{PC}}=126.8 \mathrm{~Hz},{ }^{2} J_{\mathrm{PC}}=17.6 \mathrm{~Hz},{ }^{2} J_{\mathrm{PC}}=\right.$ $\left.17.6 \mathrm{~Hz},{ }^{3} J_{\mathrm{PC}}=3.7 \mathrm{~Hz}, 3-\underline{\mathrm{C}}\right), 42.9\left(\mathrm{dddd},{ }^{1} J_{\mathrm{PC}}=139.6 \mathrm{~Hz},{ }^{2} J_{\mathrm{PC}}=5.8 \mathrm{~Hz},{ }^{3} J_{\mathrm{PC}}=5.8 \mathrm{~Hz}\right.$, $\left.{ }^{4} J_{\mathrm{PC}}=2.9 \mathrm{~Hz}, 2-\underline{\mathrm{C}}\right), 16.9-16.6\left(\mathrm{~m}, \underline{\mathrm{CH}}_{3}\right) ;{ }^{31} \mathrm{P} \mathrm{NMR}\left(162 \mathrm{MHz}, \mathrm{CDCl}_{3}, 293 \mathrm{~K}, \mathrm{ppm}\right) \delta$ $23.5\left(\mathrm{ddd},{ }^{3} J_{\mathrm{PP}}=84.5 \mathrm{~Hz},{ }^{3} J_{\mathrm{PP}}=9.3 \mathrm{~Hz},{ }^{4} J_{\mathrm{PP}}=4.5 \mathrm{~Hz}, 3-\mathrm{P}\right), 22.2\left(\mathrm{~d},{ }^{3} J_{\mathrm{PP}}=84.5 \mathrm{~Hz}\right.$, 2-P), $8.7\left(\mathrm{dd},{ }^{3} J_{\mathrm{PP}}=15.0 \mathrm{~Hz},{ }^{3} J_{\mathrm{PP}}=4.5 \mathrm{~Hz}, 4-\mathrm{P}\right), 6.7\left(\mathrm{dd},{ }^{3} J_{\mathrm{PP}}=15.0 \mathrm{~Hz},{ }^{3} J_{\mathrm{PP}}=9.3 \mathrm{~Hz}\right.$, 5-P); UV-Vis (methanol) $\lambda_{\max }$ ( $) 304$ (4300), 265 (4000) nm; IR (NaCl) 1244 ( $v(\mathrm{PO})$ ), $1022(v($ POEt $)) \mathrm{cm}^{-1}$; FT-ICR-MS (ESI, positive) Found: $m / z$ 631.1419. Calcd for $\mathrm{C}_{20} \mathrm{H}_{43} \mathrm{O}_{12} \mathrm{P}_{4} \mathrm{~S}^{+}: 631.1420\left([\mathrm{M}+\mathrm{H}]^{+}\right)$; Anal. Calcd for $\mathrm{C}_{20} \mathrm{H}_{42} \mathrm{O}_{12} \mathrm{P}_{4} \mathrm{~S} \bullet \mathrm{H}_{2} \mathrm{O}: \mathrm{C}, 37.04 ; \mathrm{H}$, 6.84; S, 4.94. Found: C, 37.55; H, 6.81; S, 4.84.

\section{2,3,4,5-Tetrakis(diethoxyphosphoryl)thiophene (6).}

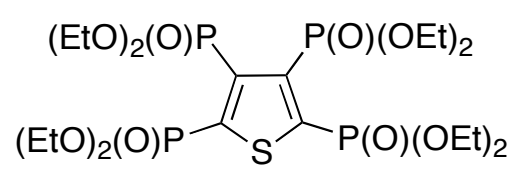

A solution of $\mathrm{mCPBA}(74.9 \mathrm{mg}, 65 \%, 0.28 \mathrm{mmol})$ in dichloromethane $(1 \mathrm{~mL})$ was added to a solution of $5(148 \mathrm{mg}, 0.235 \mathrm{mmol})$ in dichloromethane $(2 \mathrm{~mL})$ at $0{ }^{\circ} \mathrm{C}$. 
The mixture was stirred at $0{ }^{\circ} \mathrm{C}$ for $1 \mathrm{~h}$ and $20{ }^{\circ} \mathrm{C}$ for $1.5 \mathrm{~d}$. The mixture, which consisted mainly of 7 , was directly submitted to column chromatography $\left(\mathrm{SiO}_{2} /\right.$ ethyl acetate, acetone) to give $6(78.5 \mathrm{mg}, 0.125 \mathrm{mmol}, 53 \%)$. 6: colorless oil; ${ }^{1} \mathrm{H}$ NMR (400 MHz, $\left.\mathrm{CDCl}_{3}, 293 \mathrm{~K}, \mathrm{ppm}\right) \delta 4.25-4.20$ (16H, m, $\underline{\mathrm{CH}}_{2}$ ), 1.40-1.34 (24H, m, $\left.\underline{\mathrm{C}}_{3}\right)$; ${ }^{13} \mathrm{C} \mathrm{NMR}\left(101 \mathrm{MHz}, \mathrm{CDCl}_{3}, 293 \mathrm{~K}, \mathrm{ppm}\right) \delta 146.1$ (dddd, ${ }^{1} J_{\mathrm{PC}}=201.0 \mathrm{~Hz},{ }^{2} J_{\mathrm{PC}}=17.6$ $\left.\mathrm{Hz},{ }^{2} J_{\mathrm{PC}}=17.6 \mathrm{~Hz},{ }^{3} J_{\mathrm{PC}}=5.8 \mathrm{~Hz}, 2,5-\underline{\mathrm{C}}\right), 140.4\left(\mathrm{dddd},{ }^{1} J_{\mathrm{PC}}=193.4 \mathrm{~Hz},{ }^{2} J_{\mathrm{PC}}=31.2 \mathrm{~Hz}\right.$, $\left.{ }^{3} J_{\mathrm{PC}}=16.5 \mathrm{~Hz},{ }^{4} J_{\mathrm{PC}}=3.6 \mathrm{~Hz}, 3,4-\underline{\mathrm{C}}\right), 64.3\left(\mathrm{~m}, \underline{\mathrm{CH}_{2}}\right), 63.6\left(\mathrm{~d},{ }^{2} J_{\mathrm{PC}}=5.9 \mathrm{~Hz}, \underline{\mathrm{CH}}_{2}\right)$, 16.7-16.6 (m, $\left.\mathrm{CH}_{2}\right)$; ${ }^{31} \mathrm{P}$ NMR $\left(162 \mathrm{MHz}, \mathrm{CDCl}_{3}, 293 \mathrm{~K}, \mathrm{ppm}\right) \delta 8.5$ (AA'XX', $\left.J_{\mathrm{AX}}+J_{\mathrm{AX}},=11.6 \mathrm{~Hz}\right), 7.7\left(\mathrm{AA}^{\prime} \mathrm{XX}^{\prime}, J_{\mathrm{AX}}+J_{\mathrm{AX}}=11.6 \mathrm{~Hz}\right)$; UV-vis (methanol) $\lambda_{\max }(\varepsilon)$ $248(5700) \mathrm{nm}$; IR $(\mathrm{NaCl}) 1259(v(\mathrm{PO})), 1022(v(\mathrm{POEt})) \mathrm{cm}^{-1}$; FT-ICR-MS (ESI, positive) Found: $m / z$ 651.1084. Calcd for $\mathrm{C}_{20} \mathrm{H}_{40} \mathrm{O}_{12} \mathrm{P}_{4} \mathrm{SNa}^{+}: 651.1083\left([\mathrm{M}+\mathrm{Na}]^{+}\right)$; Anal. Calcd for $\mathrm{C}_{20} \mathrm{H}_{40} \mathrm{O}_{12} \mathrm{P}_{4} \mathrm{~S} \cdot 2 \mathrm{C}_{2} \mathrm{H}_{5} \mathrm{OH} \cdot \mathrm{CHCl}_{3}$ : C, 35.75; H, 6.36; S, 3.82. Found: $\mathrm{C}$, $35.38 ; \mathrm{H}, 6.61 ; \mathrm{S}, 3.46$.

\section{2,3,4,5-Tetrakis(diethoxyphosphoryl)-trans-2,3-dihydrothiophene $S$-oxide (7).}<smiles>CCOC[Pb]C1=C(POCC)[C@H](POCC)C(POCC)[S]1O</smiles>

A solution of $5(104 \mathrm{mg}, 0.165 \mathrm{mmol})$ in dichloromethane $(2 \mathrm{~mL})$ was added to a solution of $m$ CPBA $(54.4 \mathrm{mg}, 65 \%, 0.205 \mathrm{mmol})$ in dichloromethane $(2 \mathrm{~mL})$ at $0{ }^{\circ} \mathrm{C}$. The mixture was stirred at $0{ }^{\circ} \mathrm{C}$ for $70 \mathrm{~min}$, warmed to room temperature, extracted with dichloromethane, washed with saturated $\mathrm{NaHCO}_{3}$ solution, and dried over anhydrous $\mathrm{MgSO}_{4}$. Drying agent was filtered and the filtrate was concentrated under reduced pressure to give crude $7(99.0 \mathrm{mg})$. 7: colorless oil; ${ }^{1} \mathrm{H}$ NMR (400 MHz, $\mathrm{CDCl}_{3}, 293$ $\mathrm{K}$, ppm) $\delta 4.43(1 \mathrm{H}, \mathrm{ddm}, J=26.1,17.7 \mathrm{~Hz}, \mathrm{C} \underline{\mathrm{H}}), 4.32-4.05\left(16 \mathrm{H}, \mathrm{m}, \mathrm{C}_{2}\right), 3.92(1 \mathrm{H}, \mathrm{t}$, $J=17.7 \mathrm{~Hz}, \mathrm{C} \underline{\mathrm{H}}), 1.37-1.22\left(24 \mathrm{H}, \mathrm{m}, \underline{\mathrm{C}}_{3}\right) ;{ }^{13} \mathrm{C} \mathrm{NMR}\left(101 \mathrm{MHz}, \mathrm{CDCl}_{3}, 293 \mathrm{~K}, \mathrm{ppm}\right)$ $\delta 152.9(\mathrm{dm}, J=173.9 \mathrm{~Hz}), 149.8(\mathrm{dm}, J=189.2 \mathrm{~Hz}), 64.25-63.50\left(8 \mathrm{x} \mathrm{d}, \underline{\mathrm{CH}}_{2}\right), 60.7$ $(\mathrm{dm}, J=137.0 \mathrm{~Hz}), 53.9(\mathrm{dt}, J=131.2,16.7 \mathrm{~Hz}), 16.30-15.90\left(\mathrm{~m}, \underline{\mathrm{CH}}_{3}\right) ;{ }^{31} \mathrm{P}$ NMR $(162$ $\left.\mathrm{MHz}, \mathrm{CDCl}_{3}, 293 \mathrm{~K}, \mathrm{ppm}\right) \delta 20.1$ (dd, $\left.J=42.0,9.2 \mathrm{~Hz}, 3-\mathrm{P}\right), 18.8$ (d, $J=42.0 \mathrm{~Hz}, 2-\mathrm{P}$ ), 7.1 (d, $J=15.5 \mathrm{~Hz}, 5-\mathrm{P}), 5.9$ (dd, $J=15.5,9.2 \mathrm{~Hz}, 4-\mathrm{P})$; IR (NaCl) 1257 (v(PO)), 1022 $(v(\mathrm{POEt})), 796(v(\mathrm{POEt})) \mathrm{cm}^{-1}$; FT-ICR-MS (ESI, positive) Found: $m / z 669.1190$. Calcd for $\mathrm{C}_{20} \mathrm{H}_{42} \mathrm{O}_{13} \mathrm{P}_{4} \mathrm{SNa}^{+}: 669.1189\left([\mathrm{M}+\mathrm{Na}]^{+}\right)$.

2,3,4,5-Tetrakis(diethoxyphosphoryl)-trans-2,3-dihydrothiophene $S, S$-dioxide (8). 


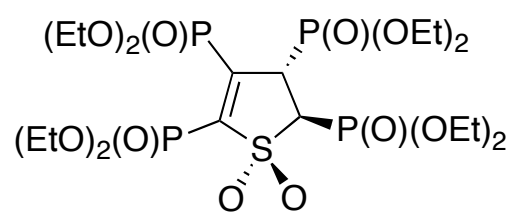

A solution of $m$ CPBA $(204 \mathrm{mg}, 65 \%, 0.77 \mathrm{mmol})$ in dichloromethane $(1 \mathrm{~mL})$ was added to $5(128 \mathrm{mg}, 0.202 \mathrm{mmol})$ at $0{ }^{\circ} \mathrm{C}$. The mixture was stirred at $0{ }^{\circ} \mathrm{C}$ for $1 \mathrm{~h}$ and $20{ }^{\circ} \mathrm{C}$ for $1 \mathrm{~d}$. The mixture was directly submitted to column chromatography ( $\mathrm{SiO}_{2} /$ ethyl acetate, acetone) to give $8(80.8 \mathrm{mg}, 0.122 \mathrm{mmol}, 60 \%)$. 8: colorless oil; ${ }^{1} \mathrm{H}$ NMR (400 MHz, $\left.\mathrm{CDCl}_{3}, 293 \mathrm{~K}, \mathrm{ppm}\right) \delta 4.30-4.14\left(18 \mathrm{H}, \mathrm{m}, \mathrm{C} \underline{\mathrm{H}}+\mathrm{C}_{2}\right), 1.35-1.19$ $\left(24 \mathrm{H}, \mathrm{m}, \mathrm{CH}_{3}\right) ;{ }^{13} \mathrm{C}$ NMR $\left(101 \mathrm{MHz}, \mathrm{CDCl}_{3}, 293 \mathrm{~K}, \mathrm{ppm}\right) \delta 147.5(\mathrm{dt}, J=183.1,9.8$ $\mathrm{Hz}, 4$ or 5-ㄷ) 145.1 (dt, J = 187.7, $11.4 \mathrm{~Hz}, 4$ or $5-\underline{\mathrm{C}}), 64.7$ (d, J=6.4 Hz, $\underline{\mathrm{CH}}_{2}$ ), 64.5 (d, $\left.J=6.6 \mathrm{~Hz}, \underline{\mathrm{CH}_{2}}\right), 64.2\left(\mathrm{~d}, J=6.0 \mathrm{~Hz}, \underline{\mathrm{CH}_{2}}\right), 64.04\left(2 \mathrm{x} \mathrm{d}, J=6.7 \mathrm{~Hz}, \underline{\mathrm{CH}}_{2}\right), 64.00(\mathrm{~d}, J$ $\left.=6.9 \mathrm{~Hz}, \underline{\mathrm{CH}}_{2}\right), 63.9\left(2 \mathrm{x} \mathrm{d}, J=6.7 \mathrm{~Hz}, \underline{\mathrm{CH}_{2}}\right), 59.0$ (dq, $\left.J=140.8,4.3 \mathrm{~Hz}, 2-\underline{\mathrm{C}}\right), 43.6$ (dddd, $J=136.4,15.3,12.0,2.6 \mathrm{~Hz}, 3-\underline{\mathrm{C}}), 16.22-15.95\left(\mathrm{~m}, \underline{\mathrm{CH}}_{3}\right) ;{ }^{31} \mathrm{P}$ NMR (162 MHz, $\left.\mathrm{CDCl}_{3}, 293 \mathrm{~K}, \mathrm{ppm}\right) \delta 19.3(\mathrm{dd}, J=38.4,9.1 \mathrm{~Hz}, 3-\mathrm{P}), 14.2$ (d, $\left.J=38.4 \mathrm{~Hz}, 2-\mathrm{P}\right), 7.2(\mathrm{~d}$, $J=15.1 \mathrm{~Hz}, 5-\mathrm{P}), 1.3(\mathrm{dd}, J=15.1,9.1 \mathrm{~Hz}, 4-\mathrm{P})$; IR $(\mathrm{NaCl}) 1335\left(v\left(\mathrm{SO}_{2}\right)\right), 1259$ ( $v(\mathrm{PO})), 1022(v(\mathrm{POEt})), 796(v(\mathrm{POEt})) \mathrm{cm}^{-1}$; FT-ICR-MS (ESI, positive) Found: $m / z$ 685.1134. Calcd for $\mathrm{C}_{20} \mathrm{H}_{42} \mathrm{O}_{14} \mathrm{P}_{4} \mathrm{SNa}^{+}: 685.1138\left([\mathrm{M}+\mathrm{Na}]^{+}\right)$; Anal. Calcd for $\mathrm{C}_{20} \mathrm{H}_{42} \mathrm{O}_{14} \mathrm{P}_{4} \mathrm{~S} \cdot \mathrm{H}_{2} \mathrm{O}$ : C, 35.30; H, 6.52; S, 4.71. Found: C, 35.66; H, 6.45; S, 4.39.

\section{2,3,4,5-Tetrakis(diethoxyphosphoryl)-trans-2,3-dihydroselenophene (9).}

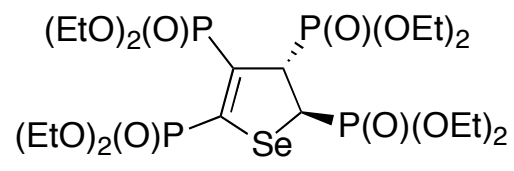

To a suspension of selenium powder (136 mg, $1.72 \mathrm{mmol})$ in ethanol ( $3 \mathrm{~mL})$ was added a solution of sodium borohydride $(81.2 \mathrm{mg}, 2.15 \mathrm{mmol})$ in ethanol $(2.5 \mathrm{~mL})$ at $0{ }^{\circ} \mathrm{C}$. The mixture was stirred for $30 \mathrm{~min}$ at $0{ }^{\circ} \mathrm{C}$, warmed to room temperature, and concentrated under reduced pressure. To sodium hydroselenide thus obtained was added ether $(5 \mathrm{~mL})$ and a solution of $1(1.11 \mathrm{~g}, 3.73 \mathrm{mmol})$ in ether $(4 \mathrm{~mL})$ at $0{ }^{\circ} \mathrm{C}$. The mixture was stirred at $0{ }^{\circ} \mathrm{C}$ for $1 \mathrm{~h}$ and $20{ }^{\circ} \mathrm{C}$ for $23 \mathrm{~h}$. The mixture was concentrated under reduced pressure, and purified by column chromatography $\left(\mathrm{SiO}_{2} /\right.$ ethyl acetate, acetone, ethanol) to give 9 (460 mg, $0.679 \mathrm{mmol}, 36 \%)$. 9: pale yellow oil; ${ }^{1} \mathrm{H}$ NMR (400 MHz, $\left.\mathrm{CDCl}_{3}, 293 \mathrm{~K}, \mathrm{ppm}\right) \delta 4.20-4.08\left(18 \mathrm{H}, \mathrm{m}, \mathrm{C} \underline{\mathrm{H}}+\mathrm{C}_{2}\right)$, $1.32-1.25\left(24 \mathrm{H}, \mathrm{m}, \underline{\mathrm{C}}_{3}\right) ;{ }^{13} \mathrm{C} \mathrm{NMR}\left(101 \mathrm{MHz}, \mathrm{CDCl}_{3}, 293 \mathrm{~K}, \mathrm{ppm}\right) \delta 146.5\left(\mathrm{dm},{ }^{1} J_{\mathrm{PC}}\right.$ $=191.4 \mathrm{~Hz}, 4-\underline{\mathrm{C}}), 134.2\left(\mathrm{dm},{ }^{1} J_{\mathrm{PC}}=191.5 \mathrm{~Hz}, 5-\underline{\mathrm{C}}\right), 64.1\left(\mathrm{~d},{ }^{2} J_{\mathrm{PC}}=6.6 \mathrm{~Hz}, \underline{\mathrm{CH}_{2}}\right), 63.6$ 
$\left(\mathrm{d},{ }^{2} J_{\mathrm{PC}}=5.9 \mathrm{~Hz}, \underline{\mathrm{CH}}_{2}\right), 63.3\left(\mathrm{~d},{ }^{2} J_{\mathrm{PC}}=5.2 \mathrm{~Hz}, \underline{\mathrm{CH}}_{2}\right), 63.2\left(\mathrm{~d},{ }^{2} J_{\mathrm{PC}}=6.6 \mathrm{~Hz}, \underline{\mathrm{CH}}_{2}\right), 63.1$ $\left(\mathrm{d},{ }^{2} J_{\mathrm{PC}}=6.7 \mathrm{~Hz}, \underline{\mathrm{CH}}_{2}\right), 63.0\left(\mathrm{~d},{ }^{2} J_{\mathrm{PC}}=5.9 \mathrm{~Hz}, \underline{\mathrm{CH}}_{2}\right), 62.7\left(\mathrm{~d},{ }^{2} J_{\mathrm{PC}}=7.2 \mathrm{~Hz}, \underline{\mathrm{CH}}_{2}\right), 62.6$ $\left(\mathrm{d},{ }^{2} J_{\mathrm{PC}}=6.0 \mathrm{~Hz}, \underline{\mathrm{CH}}_{2}\right), 55.8\left(\mathrm{dm},{ }^{1} J_{\mathrm{PC}}=119.3 \mathrm{~Hz}, 3-\underline{\mathrm{C}}\right), 34.9\left(\mathrm{dm},{ }^{1} J_{\mathrm{PC}}=129.7 \mathrm{~Hz}\right.$, 2-ㄷ), 16.4-16.1 (m, $\left.\underline{\mathrm{CH}}_{3}\right) ;{ }^{31} \mathrm{P} \mathrm{NMR}\left(162 \mathrm{MHz}, \mathrm{CDCl}_{3}, 293 \mathrm{~K}, \mathrm{ppm}\right) \delta 23.9\left(\mathrm{~d},{ }^{3} J_{\mathrm{PP}}=\right.$ 83.1 Hz, 2-P), $23.3\left(\mathrm{dd},{ }^{3} J_{\mathrm{PP}}=83.1 \mathrm{~Hz},{ }^{3} J_{\mathrm{PP}}=9.5 \mathrm{~Hz}, 3-\mathrm{P}\right), 9.3\left(\mathrm{~d},{ }^{3} J_{\mathrm{PP}}=16.8 \mathrm{~Hz}, 5-\mathrm{P}\right)$, $8.7\left(\mathrm{dd},{ }^{3} J_{\mathrm{PP}}=16.8 \mathrm{~Hz},{ }^{3} J_{\mathrm{PP}}=9.5 \mathrm{~Hz}, 4-\mathrm{P}\right) ;{ }^{77} \mathrm{Se} \mathrm{NMR}\left(76.3 \mathrm{MHz}, \mathrm{CDCl}_{3}, 295 \mathrm{~K}, \mathrm{ppm}\right)$ $\delta 555.2\left(\mathrm{~d}, J_{\mathrm{SeP}}=48.3 \mathrm{~Hz}\right) ;{ }^{77} \mathrm{Se} \mathrm{NMR}\left(76.3 \mathrm{MHz}, \mathrm{CDCl}_{3}, 295 \mathrm{~K}, \mathrm{ppm}\right.$, non ${ }^{1} \mathrm{H}$-decoupled) $\delta 555.2\left(\mathrm{dd}, J_{\mathrm{SeP}}=48.3 \mathrm{~Hz}, J_{\mathrm{SeH}}=21.2 \mathrm{~Hz}\right.$ ); UV-Vis (methanol) $\lambda_{\max }(\varepsilon)$ 321 (3600), 275 (4100) nm; IR (NaCl) 1248 (v(PO)), 1022 (v(POEt)) cm ${ }^{-1}$; FT-ICR-MS (ESI, positive) Found: $m / z$ 701.0682. Calcd for $\mathrm{C}_{20} \mathrm{H}_{42} \mathrm{O}_{12} \mathrm{P}_{4} \mathrm{SeNa}^{+}$: $701.0690\left([\mathrm{M}+\mathrm{Na}]^{+}\right)$; Anal. Calcd for $\mathrm{C}_{20} \mathrm{H}_{42} \mathrm{O}_{12} \mathrm{P}_{4} \mathrm{Se}: \mathrm{C}, 35.46 ; \mathrm{H}, 6.25$. Found: $\mathrm{C}$, $35.74 ; \mathrm{H}, 6.41$.

\section{2,3,4,5-Tetrakis(diethoxyphosphoryl)selenophene (10).}

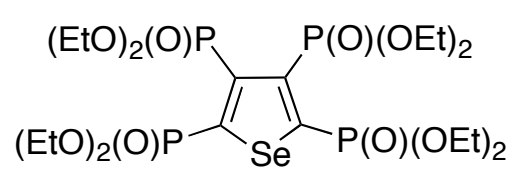

A solution of $m$ CPBA $(42.9 \mathrm{mg}, 65 \%, 0.16 \mathrm{mmol})$ in dichloromethane $(1 \mathrm{~mL})$ was added to a solution of $9(104 \mathrm{mg}, 0.154 \mathrm{mmol})$ in dichloromethane $(2 \mathrm{~mL})$ at $0{ }^{\circ} \mathrm{C}$. The mixture was stirred at $0{ }^{\circ} \mathrm{C}$ for $1 \mathrm{~h}$ and $20^{\circ} \mathrm{C}$ for $22 \mathrm{~h}$. The mixture was directly submitted to column chromatography ( $\mathrm{SiO}_{2} /$ ethyl acetate, acetone, ethanol) to give 9 (70.5 mg, $0.104 \mathrm{mmol}, 68 \%$ ). 10: colorless oil; ${ }^{1} \mathrm{H} \mathrm{NMR}\left(400 \mathrm{MHz}, \mathrm{CDCl}_{3}, 293 \mathrm{~K}\right.$, ppm) $\delta 3.21-4.15\left(16 \mathrm{H}, \mathrm{m}, \underline{\mathrm{C}}_{2}\right), 1.34-1.30\left(24 \mathrm{H}, \mathrm{m}, \mathrm{C}_{3}\right) ;{ }^{13} \mathrm{C} \mathrm{NMR}(101 \mathrm{MHz}$, $\left.\mathrm{CDCl}_{3}, 293 \mathrm{~K}, \mathrm{ppm}\right) \delta 154.9\left(\mathrm{dm},{ }^{1} J_{\mathrm{PC}}=195.8 \mathrm{~Hz}, 2,5-\underline{\mathrm{C}}\right), 142.0\left(\mathrm{dm},{ }^{1} J_{\mathrm{PC}}=184.8 \mathrm{~Hz}\right.$, $3,4-\underline{\mathrm{C}}), 63.6\left(\mathrm{~d},{ }^{2} J_{\mathrm{PC}}=5.9 \mathrm{~Hz} \underline{\mathrm{CH}} \mathrm{H}_{2}\right), 63.0\left(\mathrm{~d},{ }^{2} J_{\mathrm{PC}}=6.6 \mathrm{~Hz}, \underline{\mathrm{CH}_{2}}\right), 16.2\left(\mathrm{~m}, \underline{\mathrm{CH}}_{3}\right) ;{ }^{31} \mathrm{P}$ NMR (162 MHz, $\left.\mathrm{CDCl}_{3}, 293 \mathrm{~K}, \mathrm{ppm}\right) \delta 10.6\left(\mathrm{AA}^{\prime} \mathrm{XX}\right.$ ', $\left.J_{\mathrm{AX}}+J_{\mathrm{AX}},=16.2 \mathrm{~Hz}\right), 10.0$ $\left(\mathrm{AA}^{\prime} \mathrm{XX}\right.$ ', $\left.J_{\mathrm{AX}}+J_{\mathrm{AX}}{ }^{\prime}=16.2 \mathrm{~Hz}\right) ;{ }^{77} \mathrm{Se} \mathrm{NMR}\left(76.3 \mathrm{MHz}, \mathrm{CDCl}_{3}, 295 \mathrm{~K}, \mathrm{ppm}\right) \delta 929.5(\mathrm{t}$, $J_{\mathrm{SeP}}=49.9 \mathrm{~Hz}$ ); UV-vis (methanol) $\lambda_{\max }(\varepsilon) 268 \mathrm{~nm}(4300)$; IR (NaCl) $1257(v(\mathrm{PO})$ ), $1022(v(\mathrm{POEt})) \mathrm{cm}^{-1}$; FT-ICR-MS (ESI, positive) Found: $\mathrm{m} / z$ 699.0530. Calcd for $\mathrm{C}_{20} \mathrm{H}_{40} \mathrm{O}_{12} \mathrm{P}_{4} \mathrm{SeNa}^{+}: 699.0533\left([\mathrm{M}+\mathrm{Na}]^{+}\right)$; Anal. Calcd for $\mathrm{C}_{20} \mathrm{H}_{40} \mathrm{O}_{12} \mathrm{P}_{4} \mathrm{Se} \cdot \mathrm{C}_{2} \mathrm{H}_{5} \mathrm{OH}: \mathrm{C}$, 36.63 ; H, 6.43\%. Found: C, 36.99; H, 6.52\%.

\section{Reference}

1) Kyba, E. P.; Rines, S. P.; Owens, P. W.; Chou, S. P. Tetrahedron Lett. 1981, 22, 1875. 


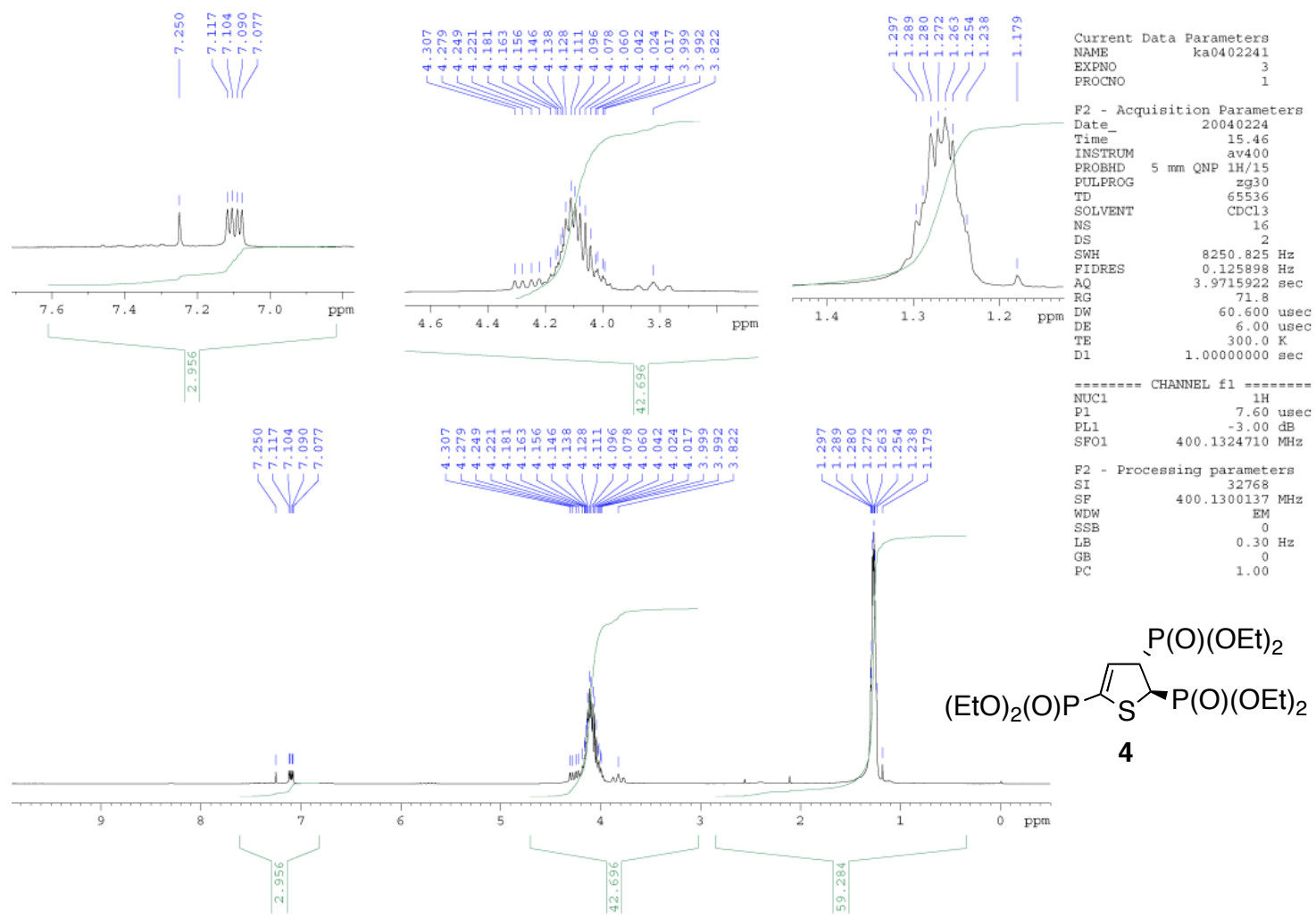

Figure 1-1. ${ }^{1} \mathrm{H}$ NMR $\left(400 \mathrm{MHz}, \mathrm{CDCl}_{3}, 293 \mathrm{~K}\right)$ of 4.

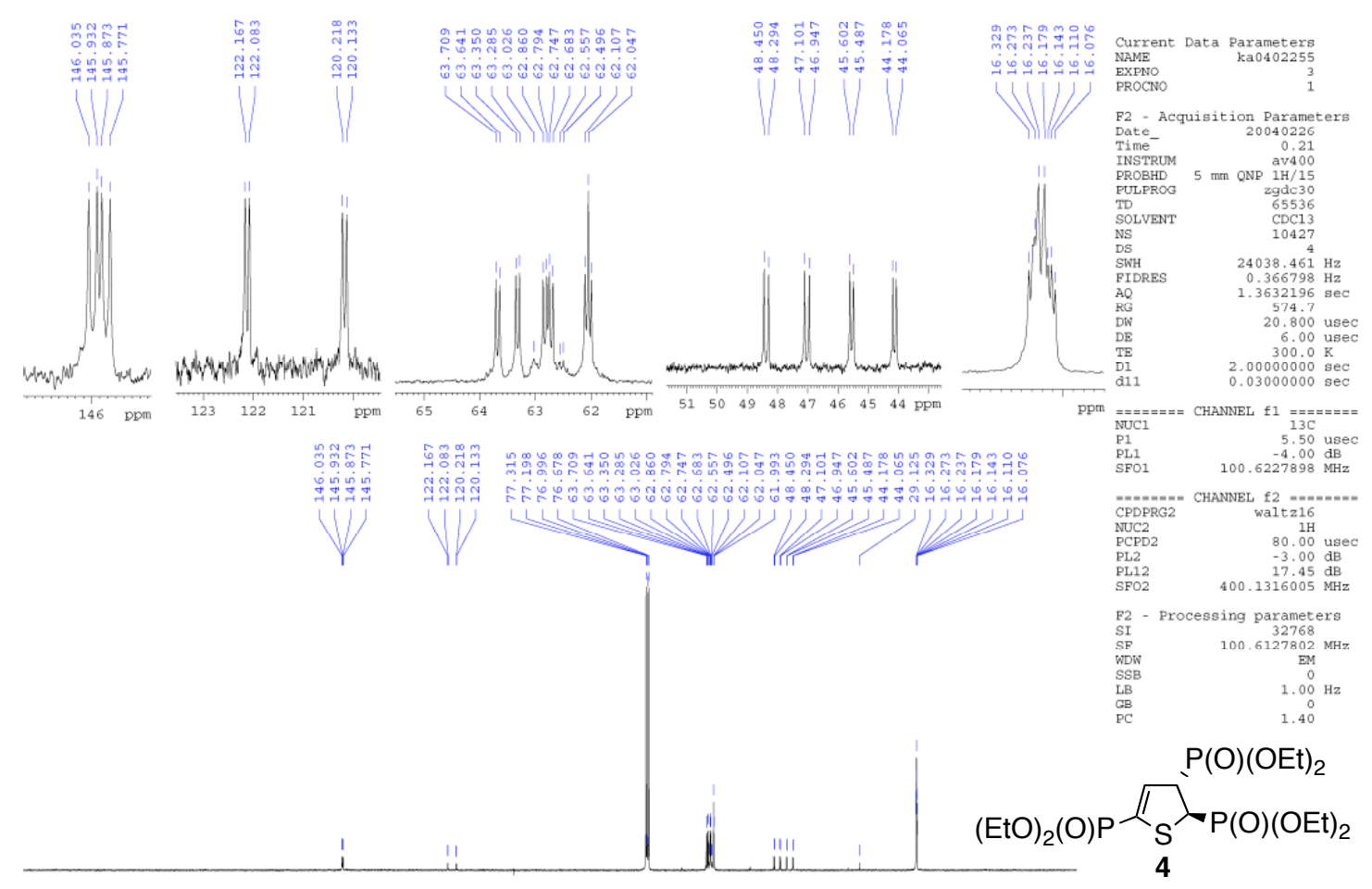

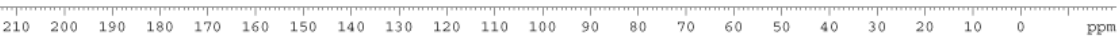

Figure 1-2. ${ }^{13} \mathrm{C}$ NMR $\left(101 \mathrm{MHz}, \mathrm{CDCl}_{3}, 293 \mathrm{~K}\right)$ of 4. 


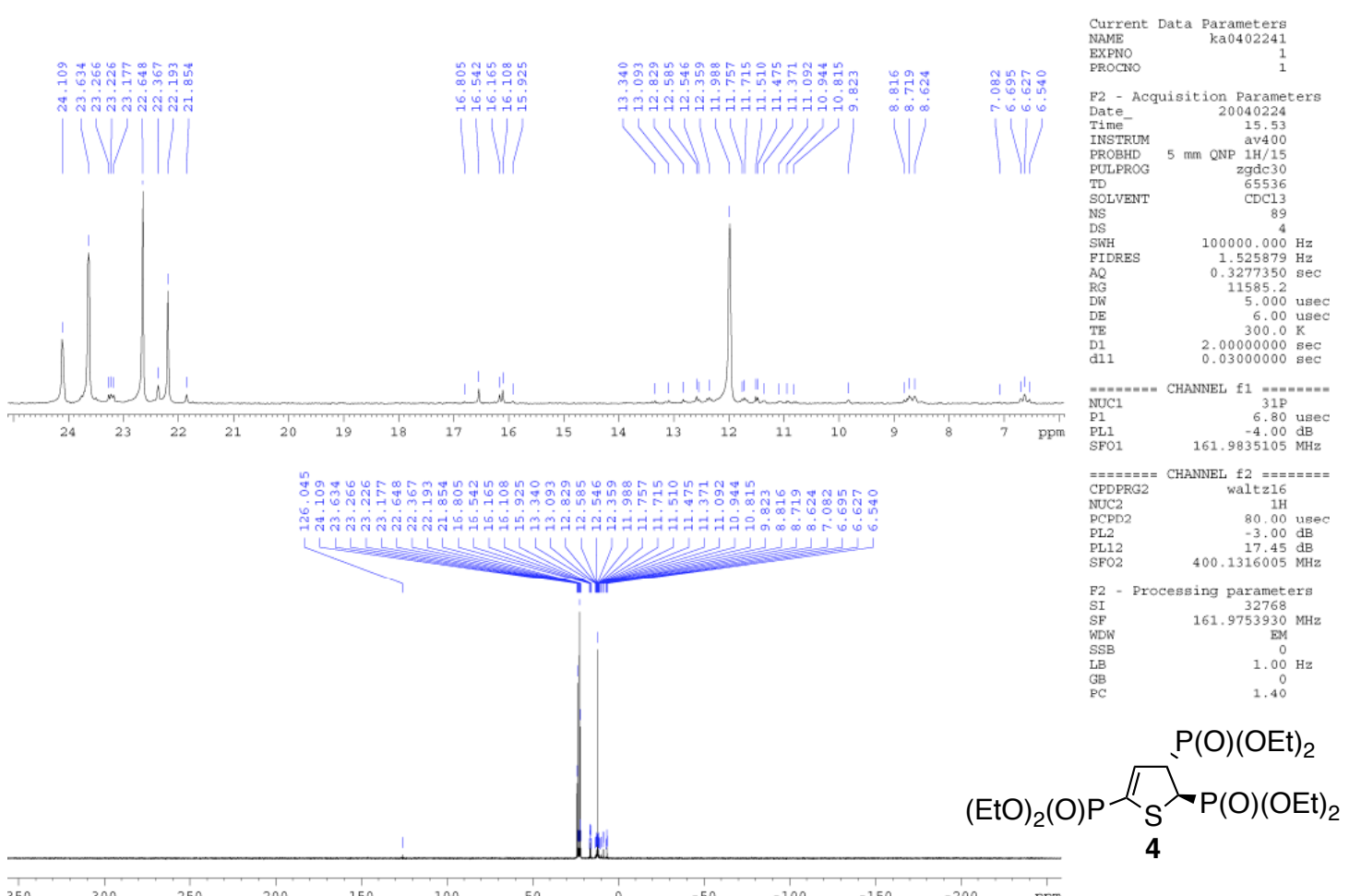

Figure 1-3. ${ }^{31} \mathrm{P}$ NMR $\left(162 \mathrm{MHz}, \mathrm{CDCl}_{3}, 293 \mathrm{~K}\right)$ of 4.

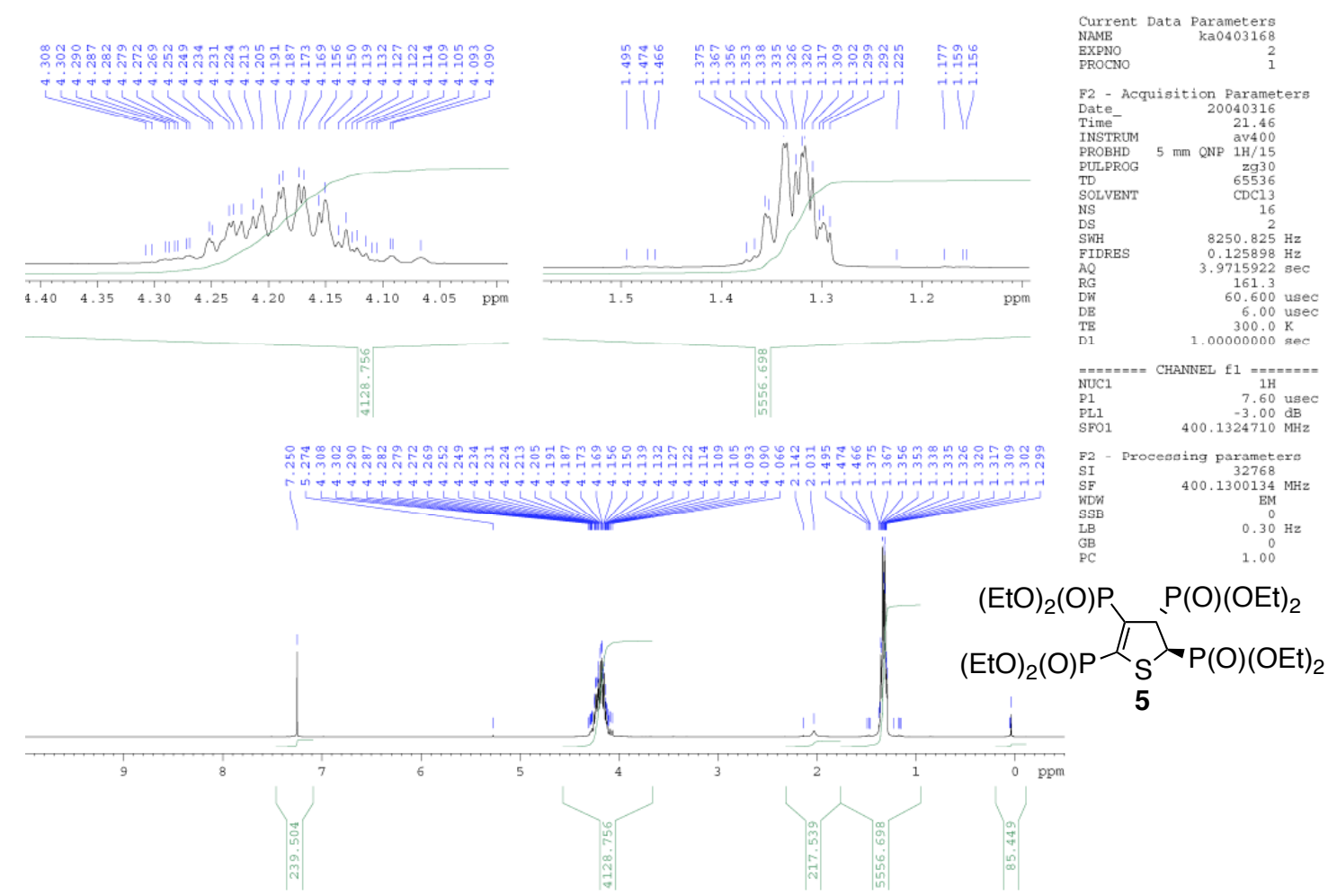

Figure 2-1. ${ }^{1} \mathrm{H}$ NMR (400 MHz, $\left.\mathrm{CDCl}_{3}, 293 \mathrm{~K}\right)$ of 5. 


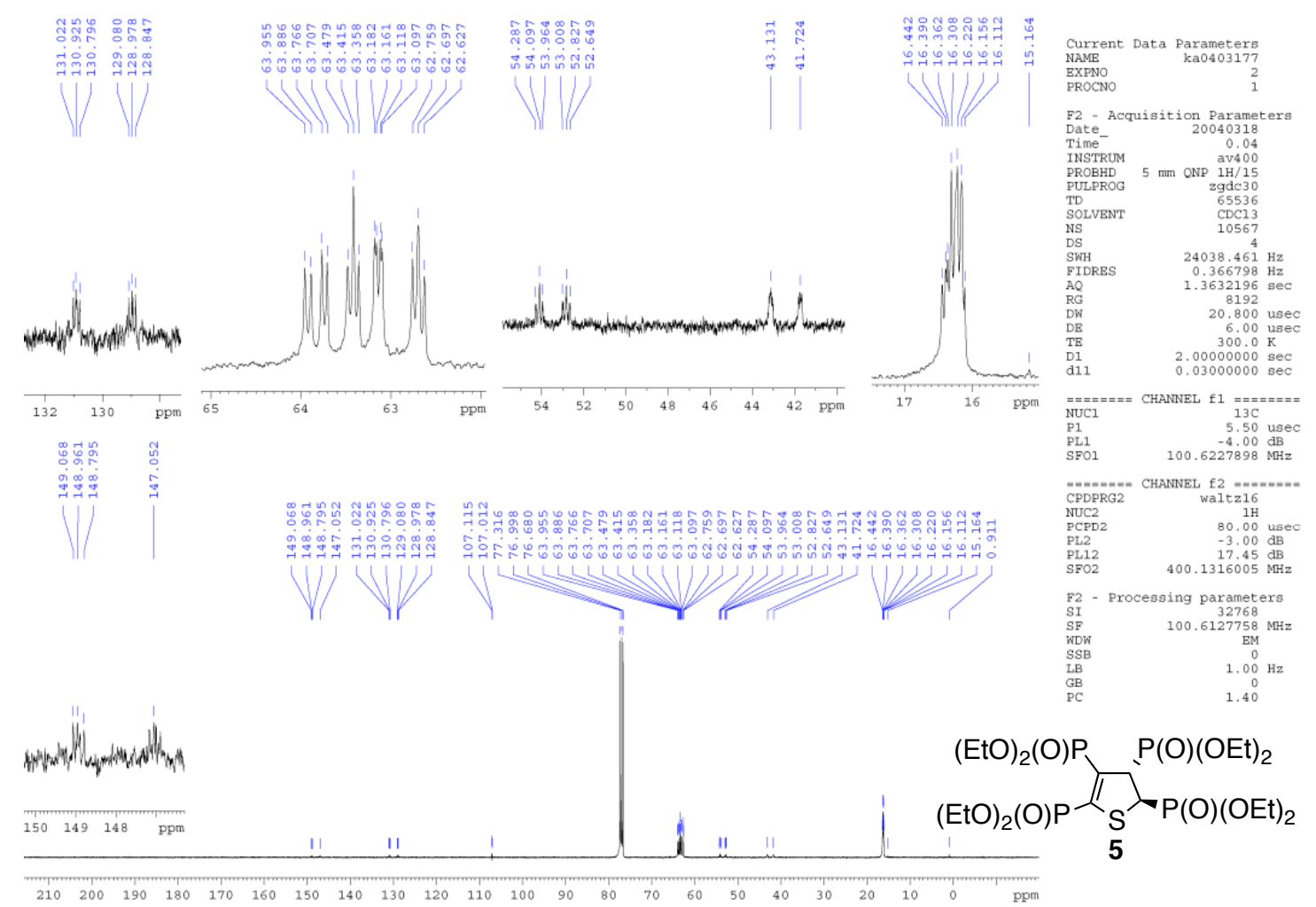

Figure 2-2. ${ }^{13} \mathrm{C} \mathrm{NMR}\left(101 \mathrm{MHz}, \mathrm{CDCl}_{3}, 293 \mathrm{~K}\right)$ of 5.
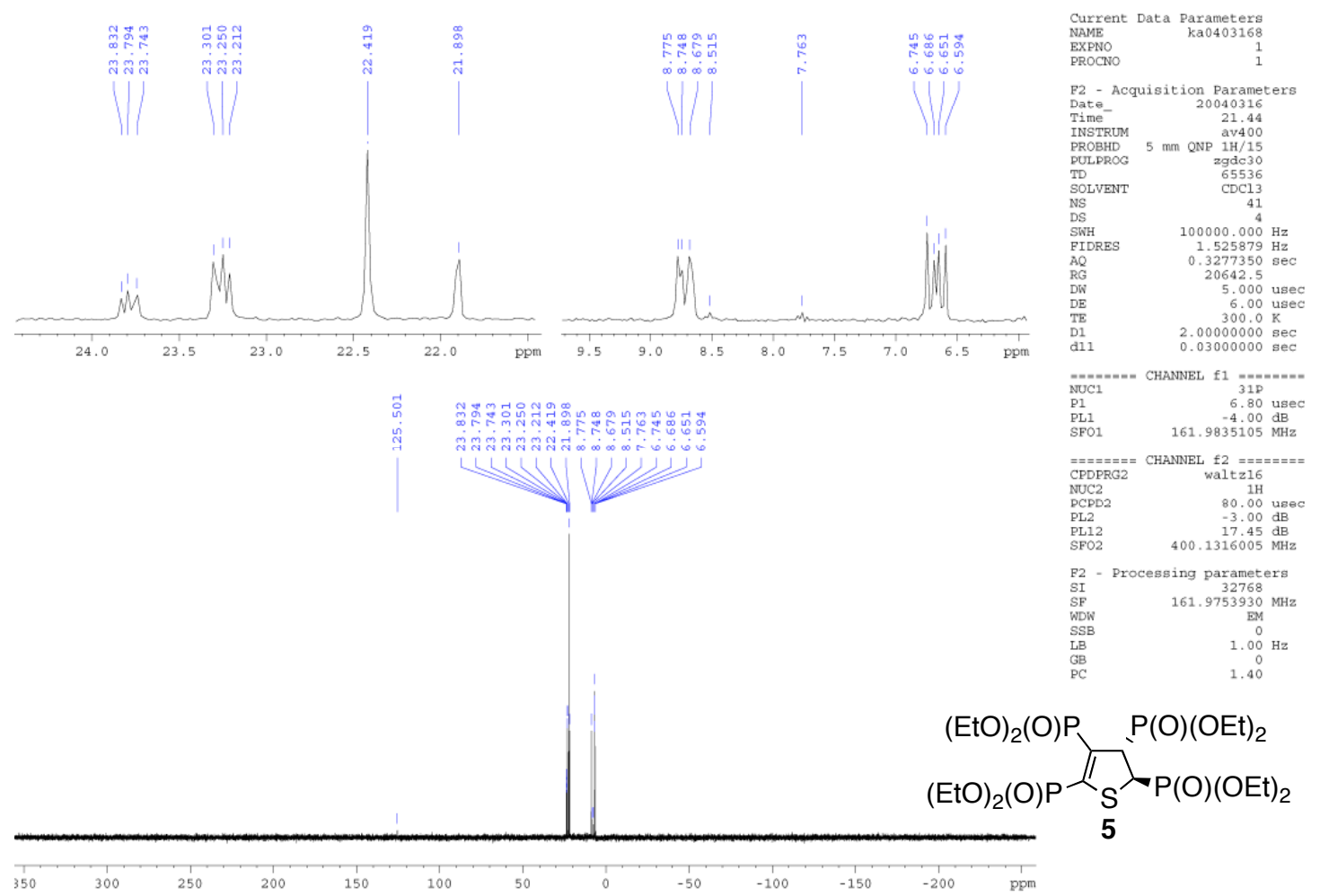

Figure 2-3. ${ }^{31} \mathrm{P}$ NMR (162 MHz, $\left.\mathrm{CDCl}_{3}, 293 \mathrm{~K}\right)$ of 5. 


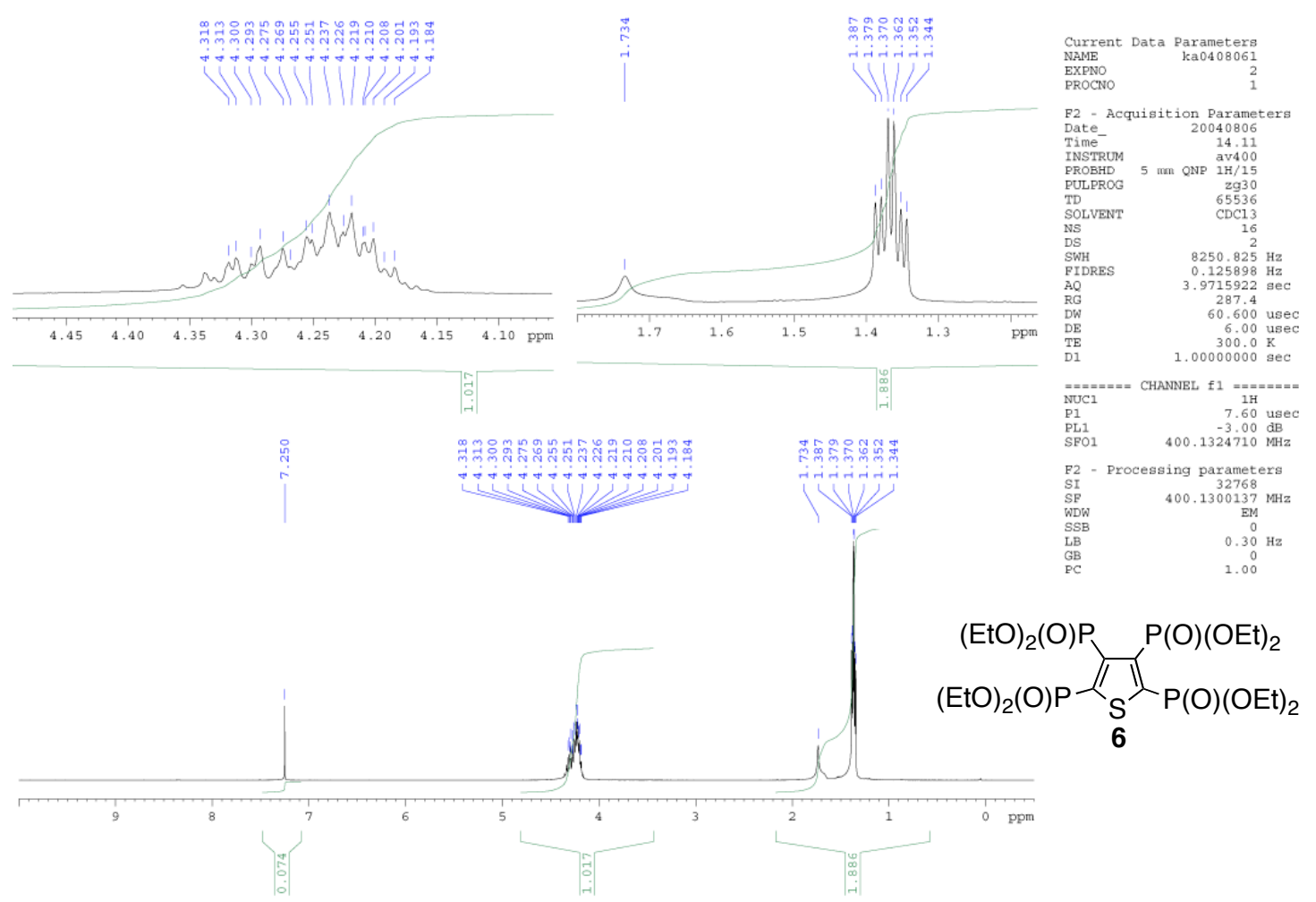

Figure 3-1. ${ }^{1} \mathrm{H}$ NMR (400 MHz, $\left.\mathrm{CDCl}_{3}, 293 \mathrm{~K}\right)$ of 6.

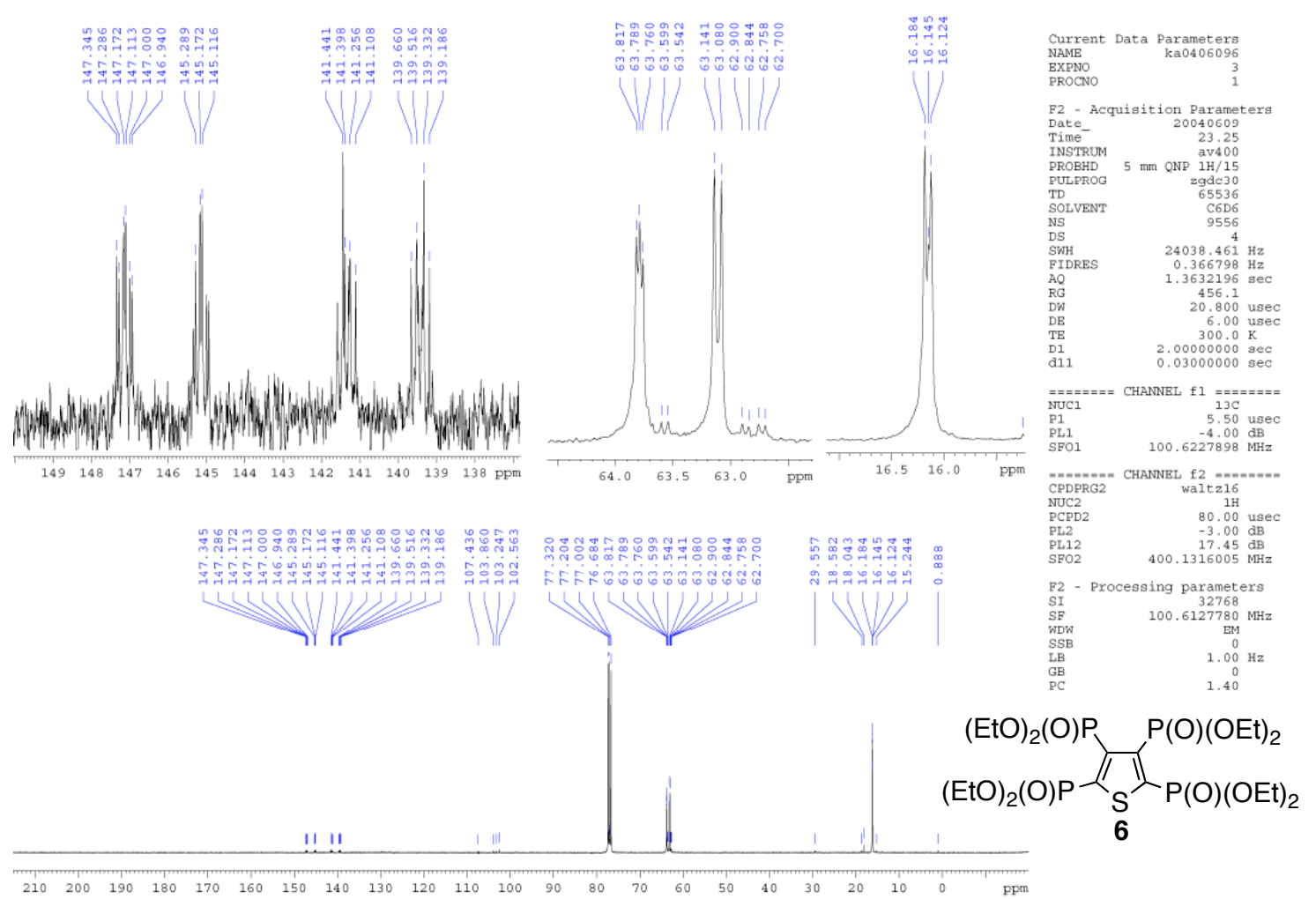

Figure 3-2. ${ }^{13} \mathrm{C}$ NMR $\left(101 \mathrm{MHz}, \mathrm{CDCl}_{3}, 293 \mathrm{~K}\right)$ of 6. 


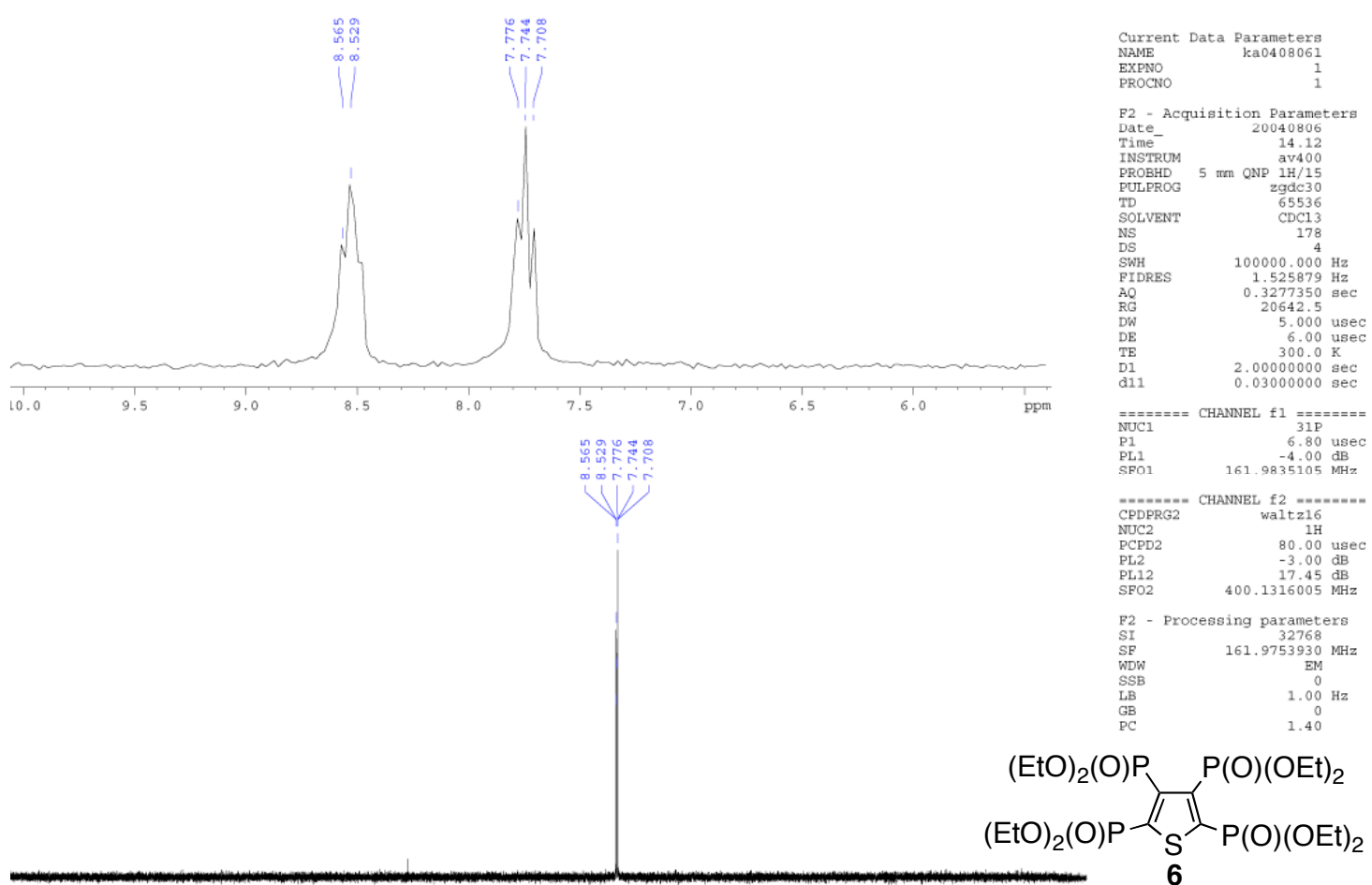

Figure 3-3. ${ }^{31} \mathrm{P}$ NMR $\left(162 \mathrm{MHz}, \mathrm{CDCl}_{3}, 293 \mathrm{~K}\right)$ of 6.
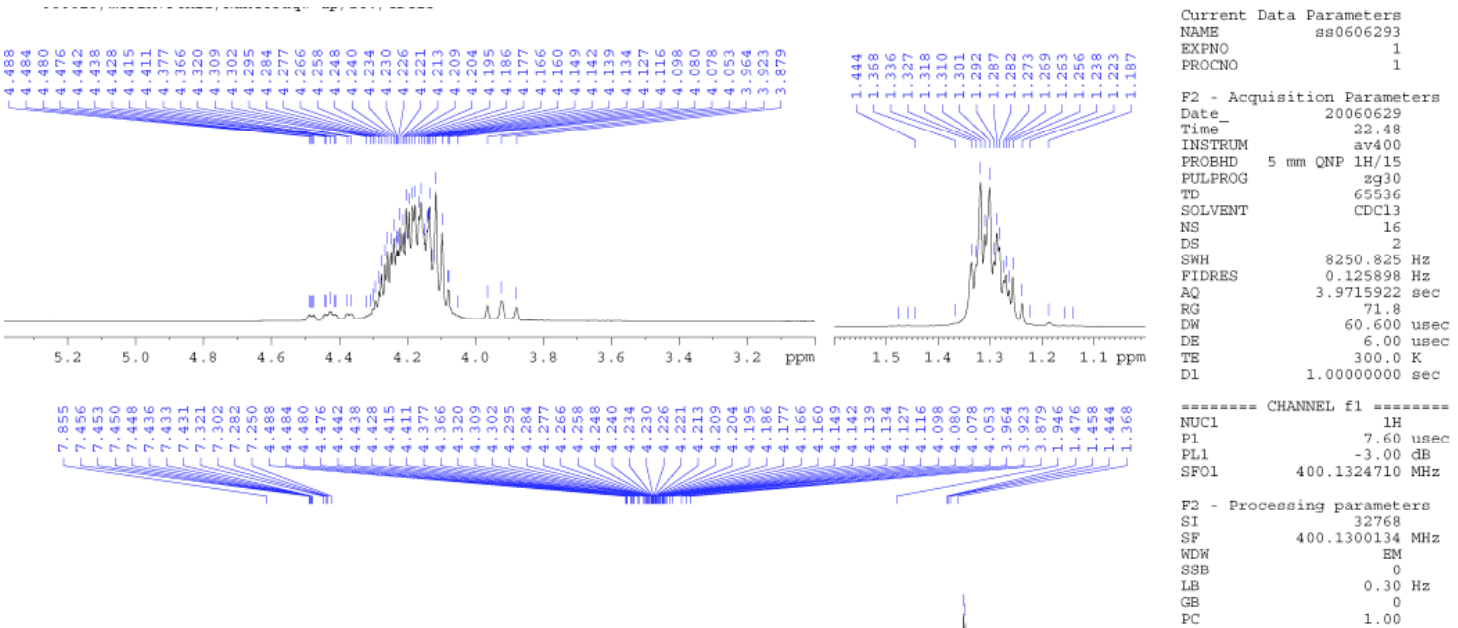

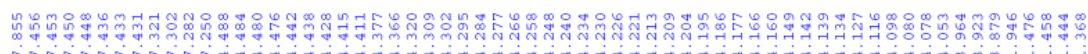
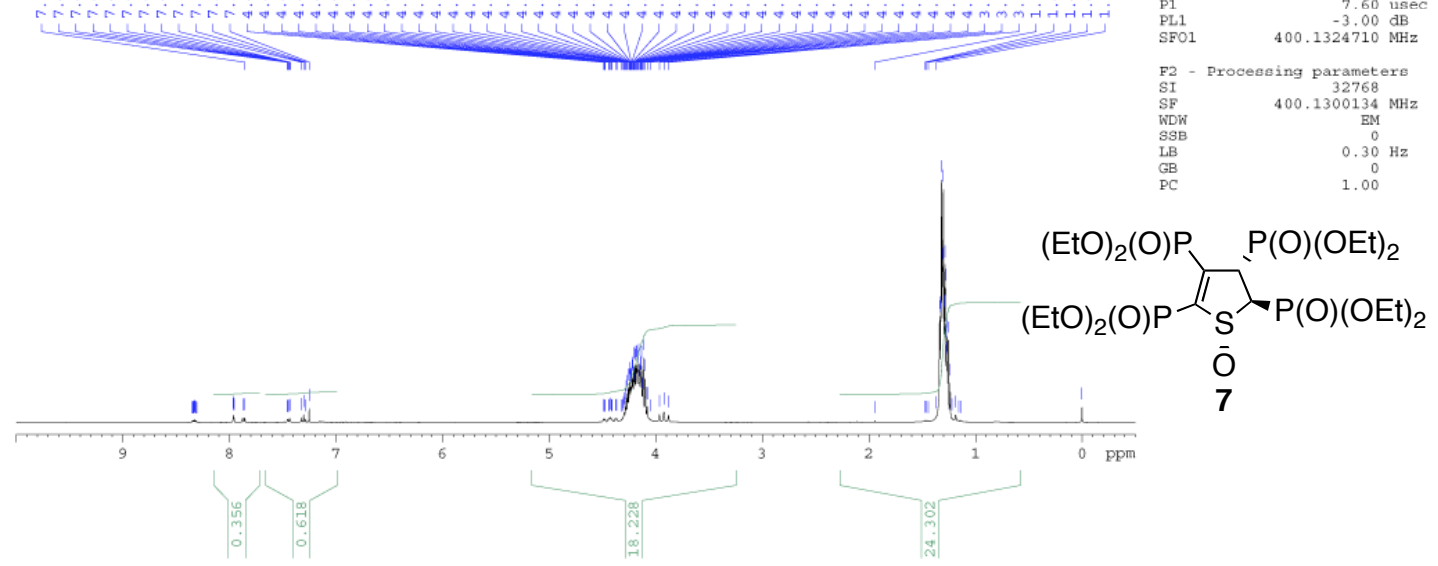

Figure 4-1. ${ }^{1} \mathrm{H}$ NMR (400 MHz, $\left.\mathrm{CDCl}_{3}, 293 \mathrm{~K}\right)$ of 7 


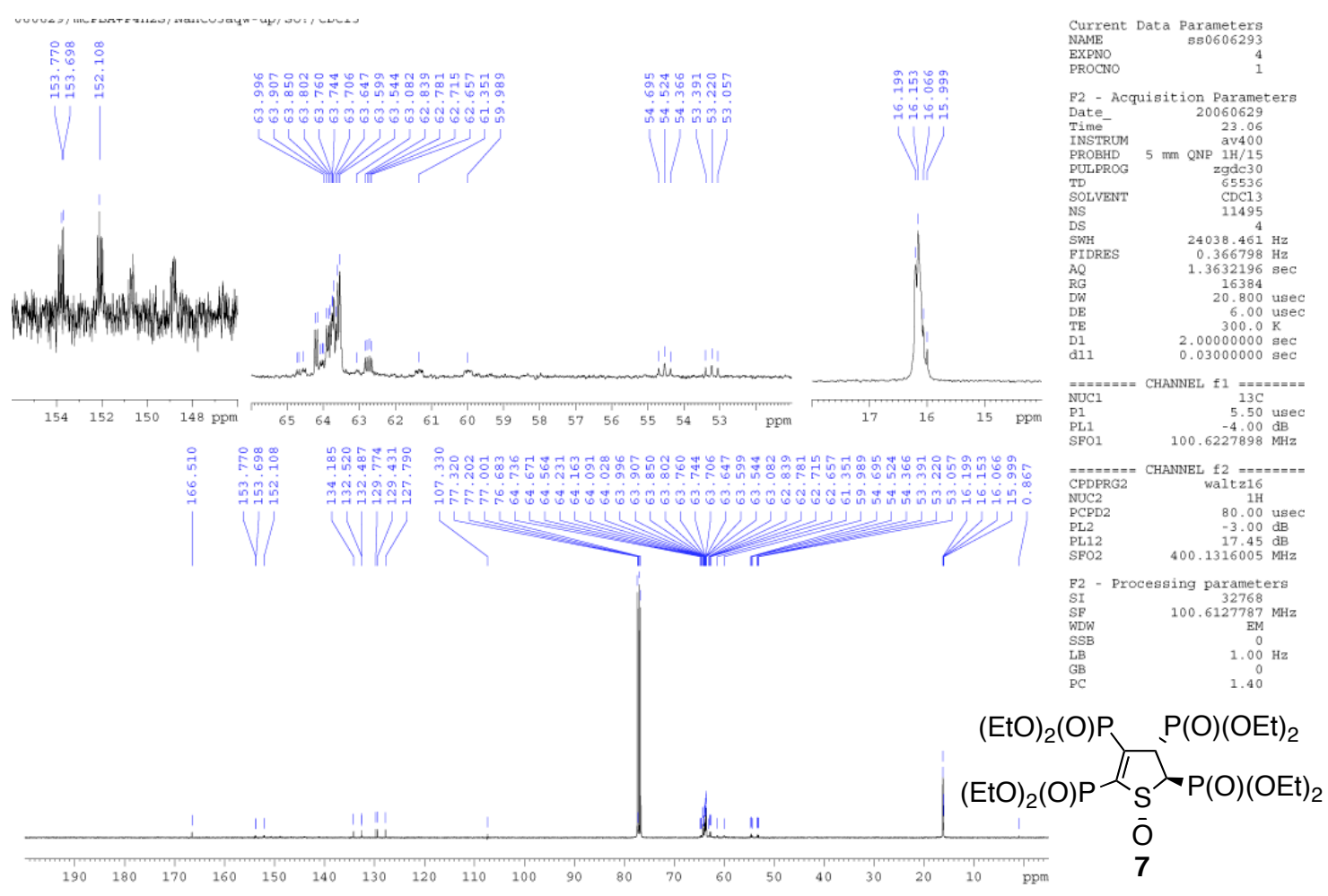

Figure 4-2. ${ }^{13} \mathrm{C}$ NMR $\left(101 \mathrm{MHz}, \mathrm{CDCl}_{3}, 293 \mathrm{~K}\right)$ of 7

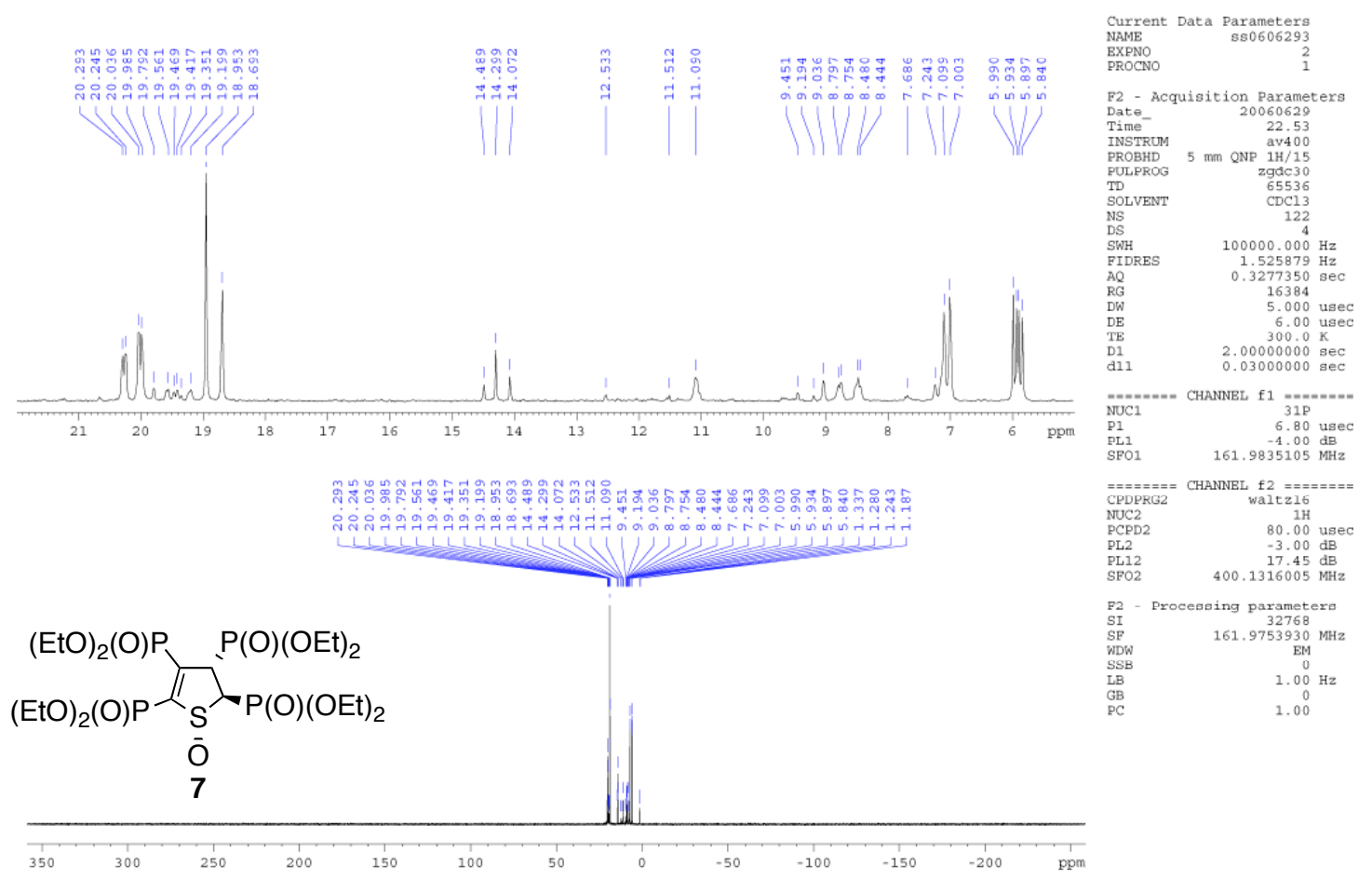

Figure 4-3. ${ }^{31} \mathrm{P}$ NMR $\left(162 \mathrm{MHz}, \mathrm{CDCl}_{3}, 293 \mathrm{~K}\right)$ of 7 


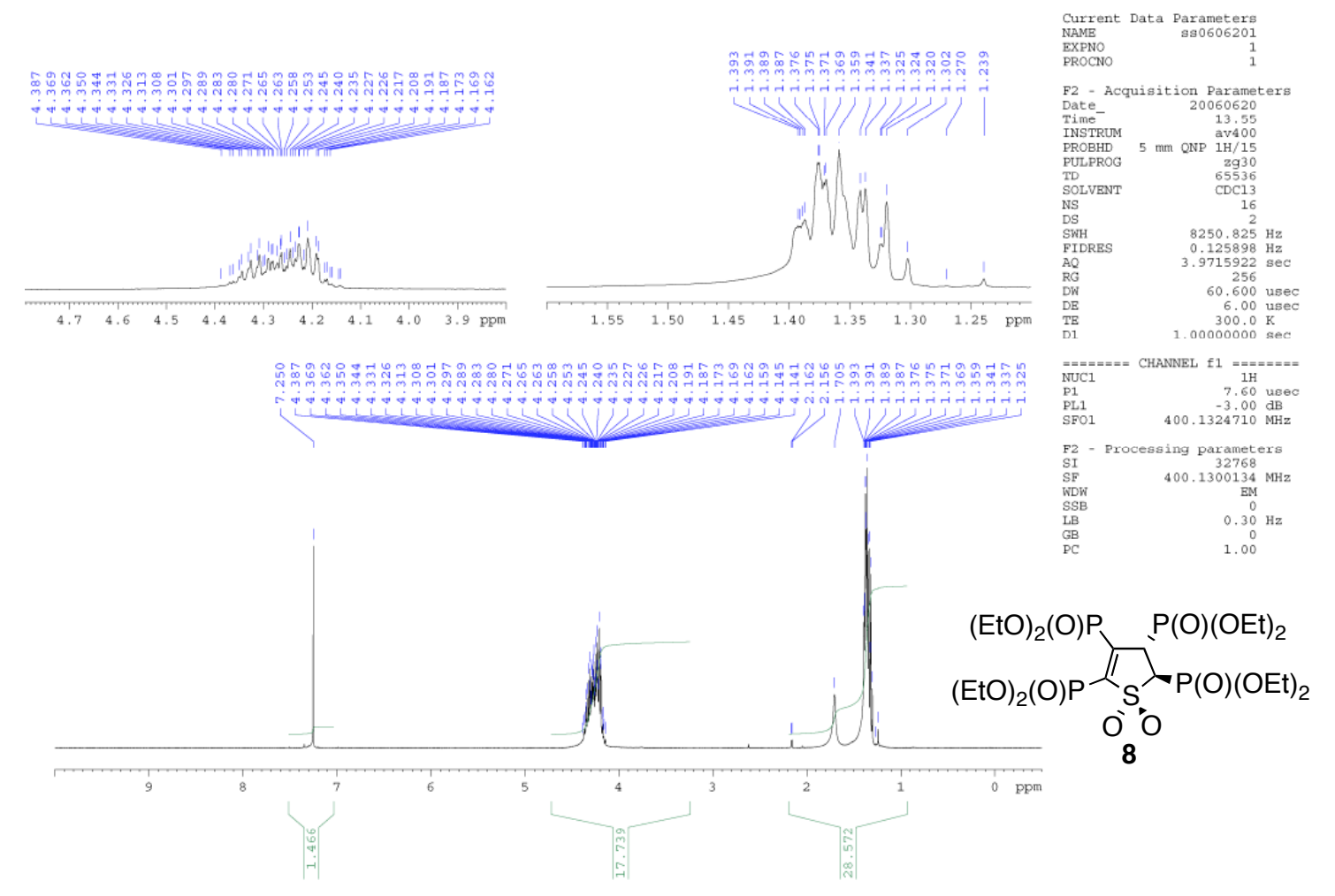

Figure 5-1. ${ }^{1} \mathrm{H} \mathrm{NMR}\left(400 \mathrm{MHz}, \mathrm{CDCl}_{3}, 293 \mathrm{~K}\right)$ of 8

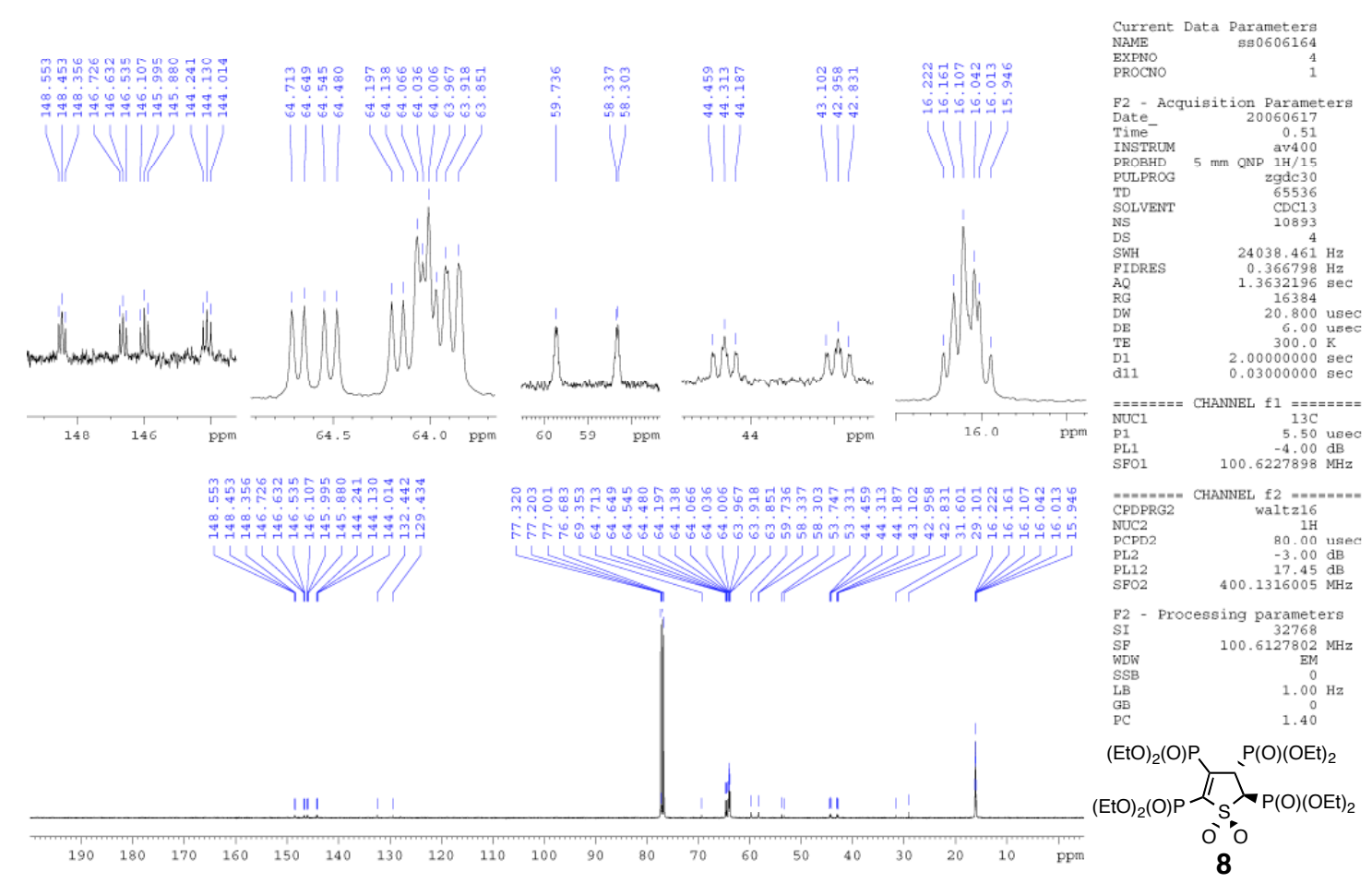

Figure 5-2. ${ }^{13} \mathrm{C}$ NMR $\left(101 \mathrm{MHz}, \mathrm{CDCl}_{3}, 293 \mathrm{~K}\right)$ of 8 


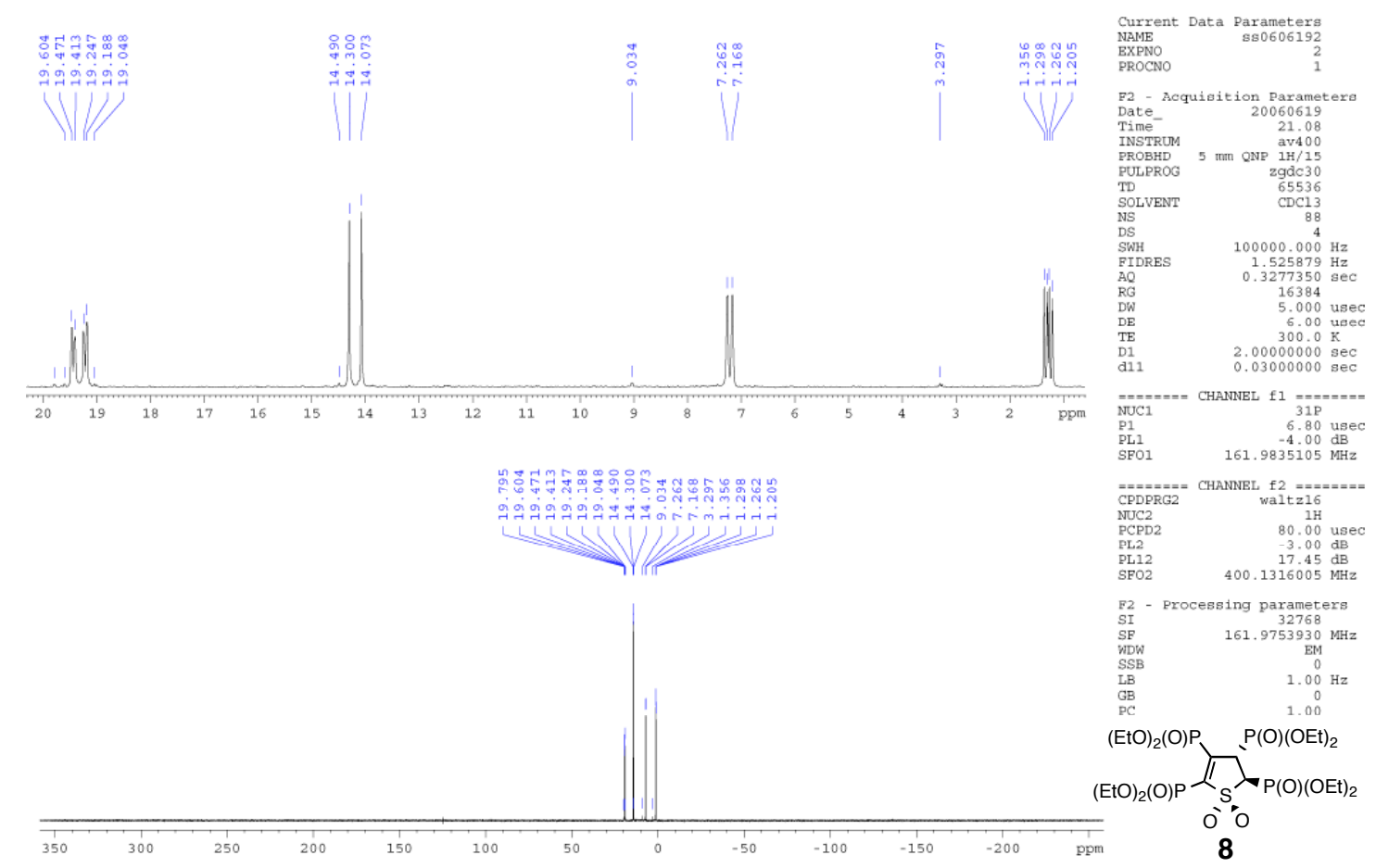

Figure 5-3. ${ }^{31} \mathrm{P} \mathrm{NMR}\left(162 \mathrm{MHz}, \mathrm{CDCl}_{3}, 293 \mathrm{~K}\right)$ of 8

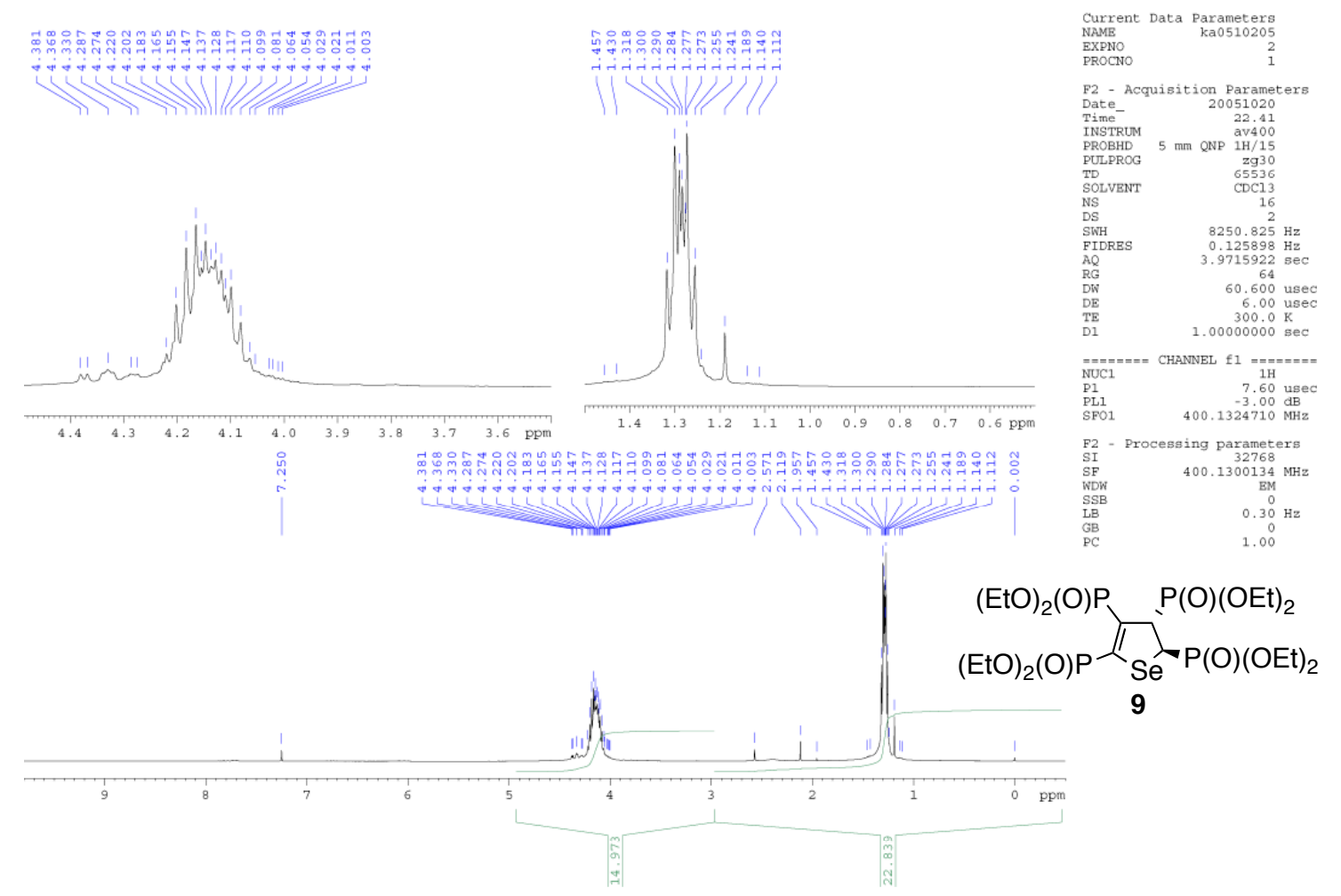

Figure 6-1. ${ }^{1} \mathrm{H}$ NMR (400 MHz, $\left.\mathrm{CDCl}_{3}, 293 \mathrm{~K}\right)$ of 9 


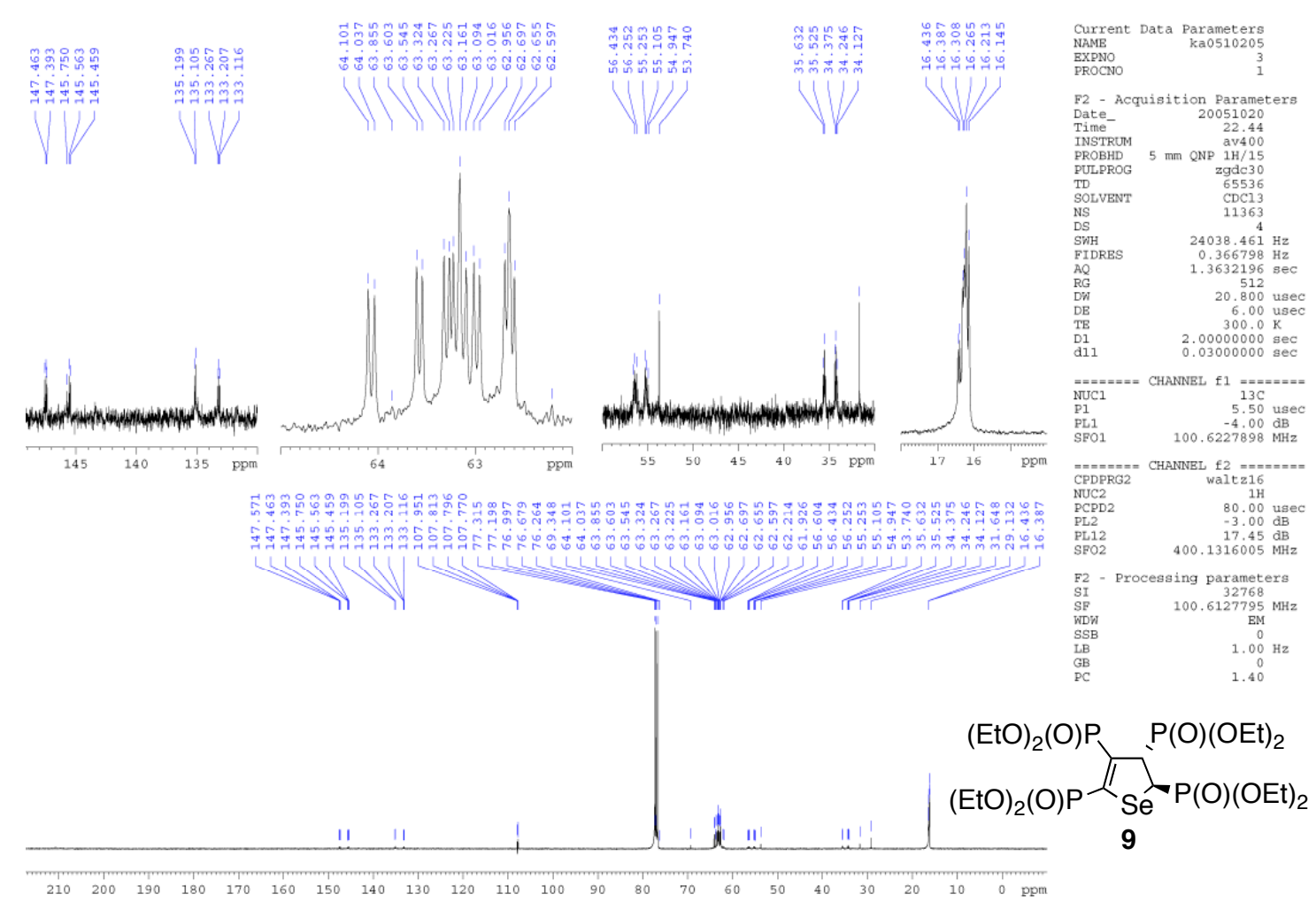

Figure 6-2. ${ }^{13} \mathrm{C}$ NMR $\left(101 \mathrm{MHz}, \mathrm{CDCl}_{3}, 293 \mathrm{~K}\right)$ of 9

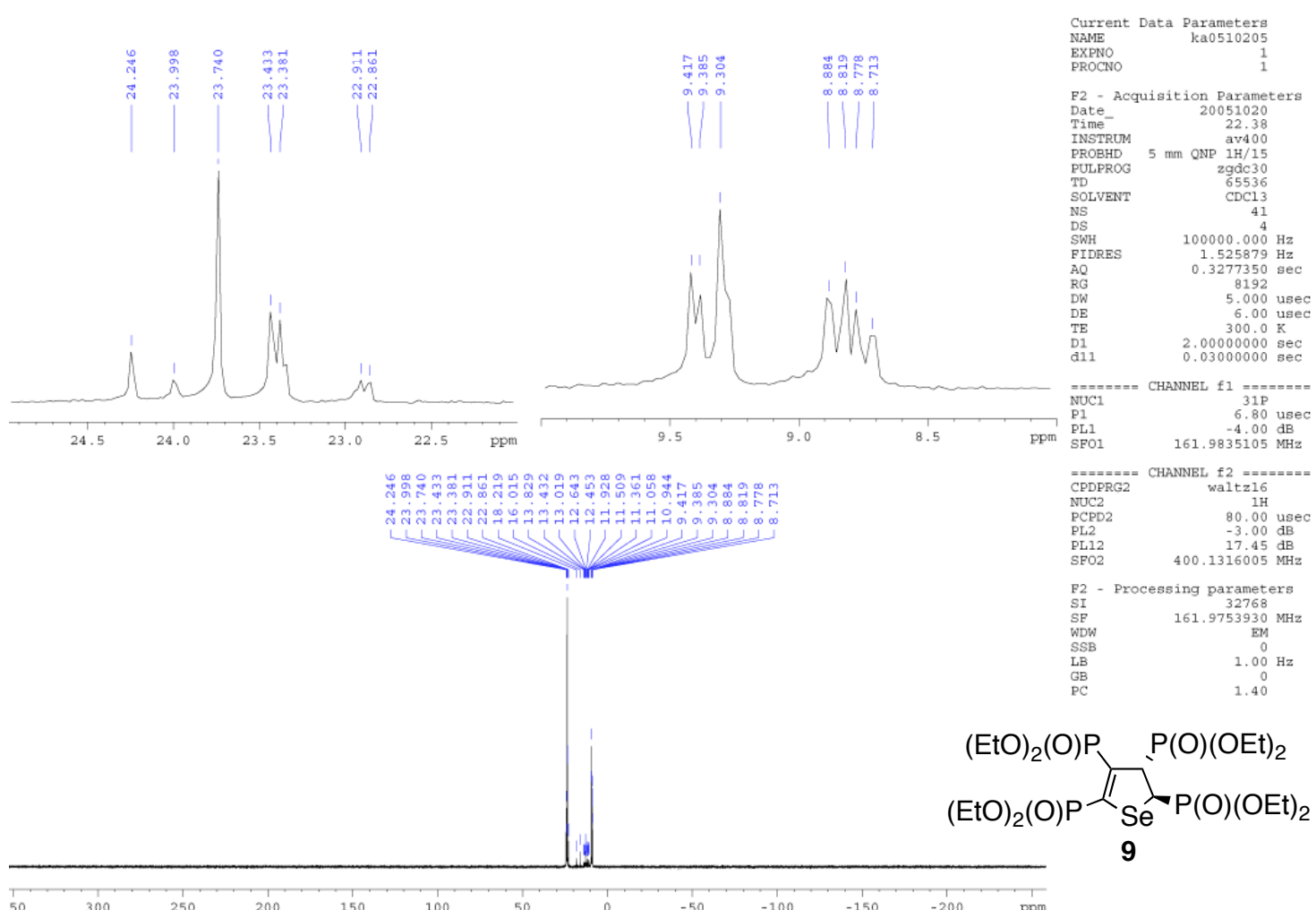

Figure 6-3. ${ }^{31} \mathrm{P}$ NMR (162 MHz, $\left.\mathrm{CDCl}_{3}, 293 \mathrm{~K}\right)$ of 9 


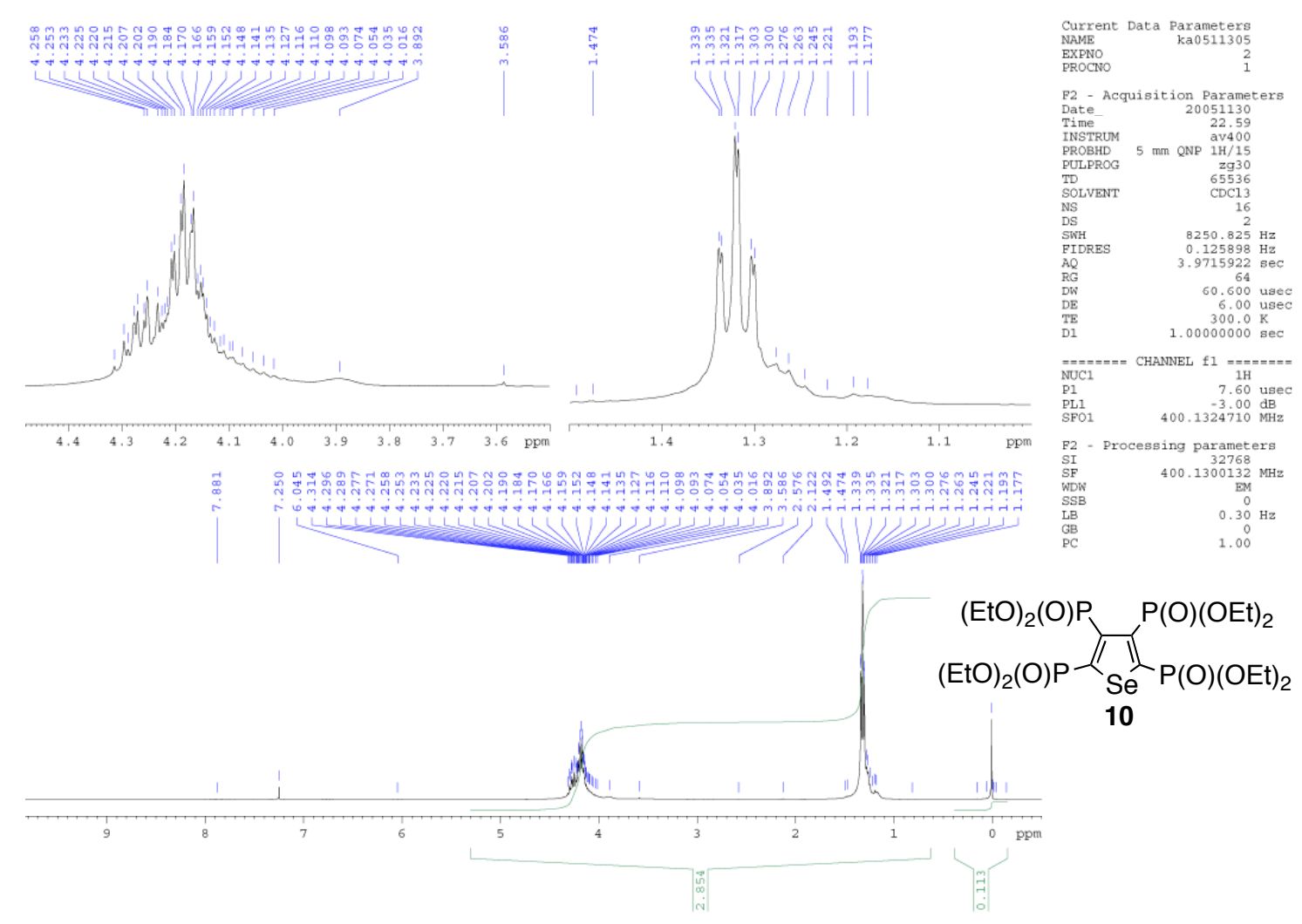

Figure 7-1. ${ }^{1} \mathrm{H}$ NMR (400 MHz, $\left.\mathrm{CDCl}_{3}, 293 \mathrm{~K}\right)$ of $\mathbf{1 0}$

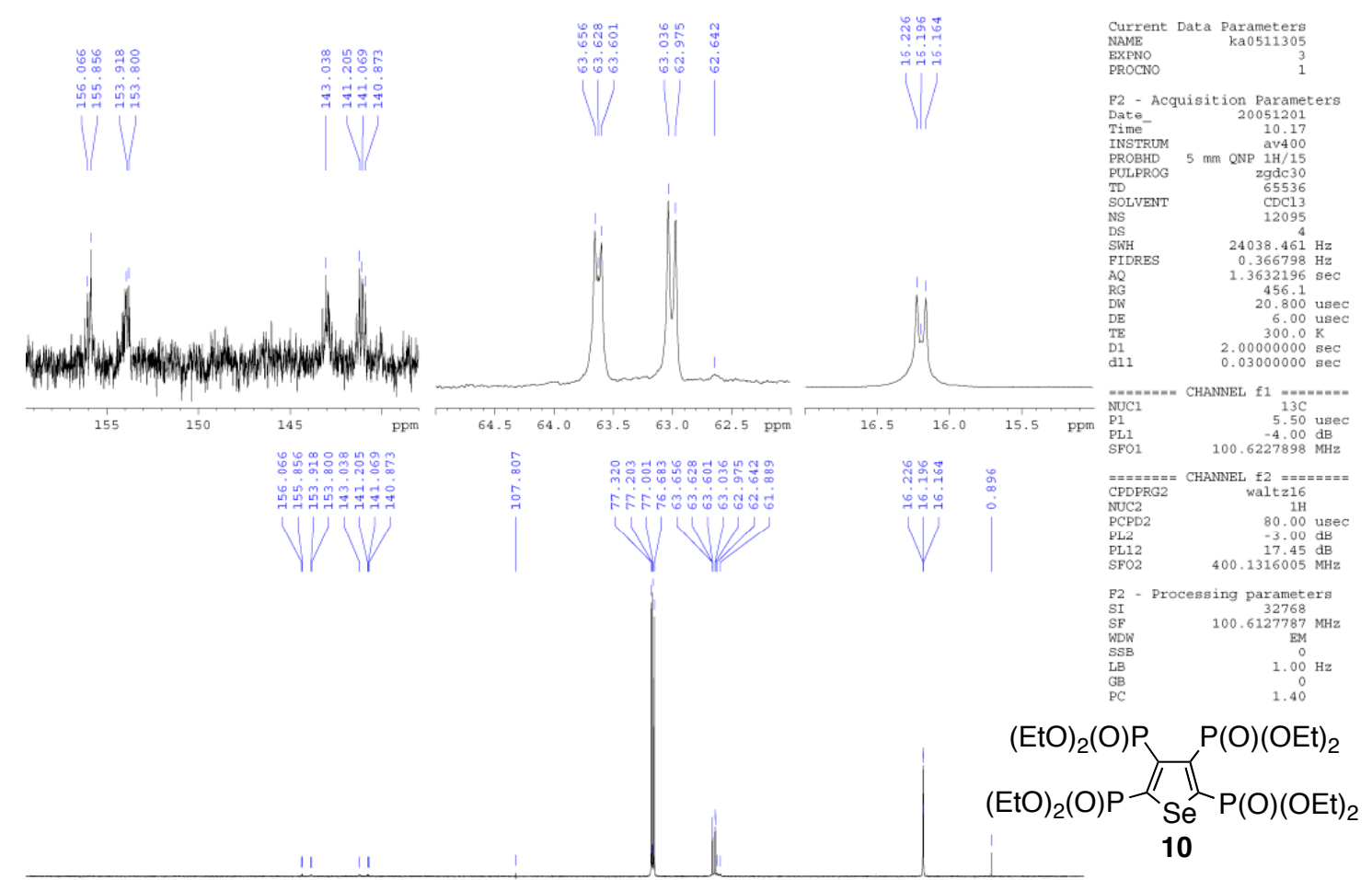

Figure 7-2. ${ }^{13} \mathrm{C} \mathrm{NMR}\left(101 \mathrm{MHz}, \mathrm{CDCl}_{3}, 293 \mathrm{~K}\right)$ of $\mathbf{1 0}$ 


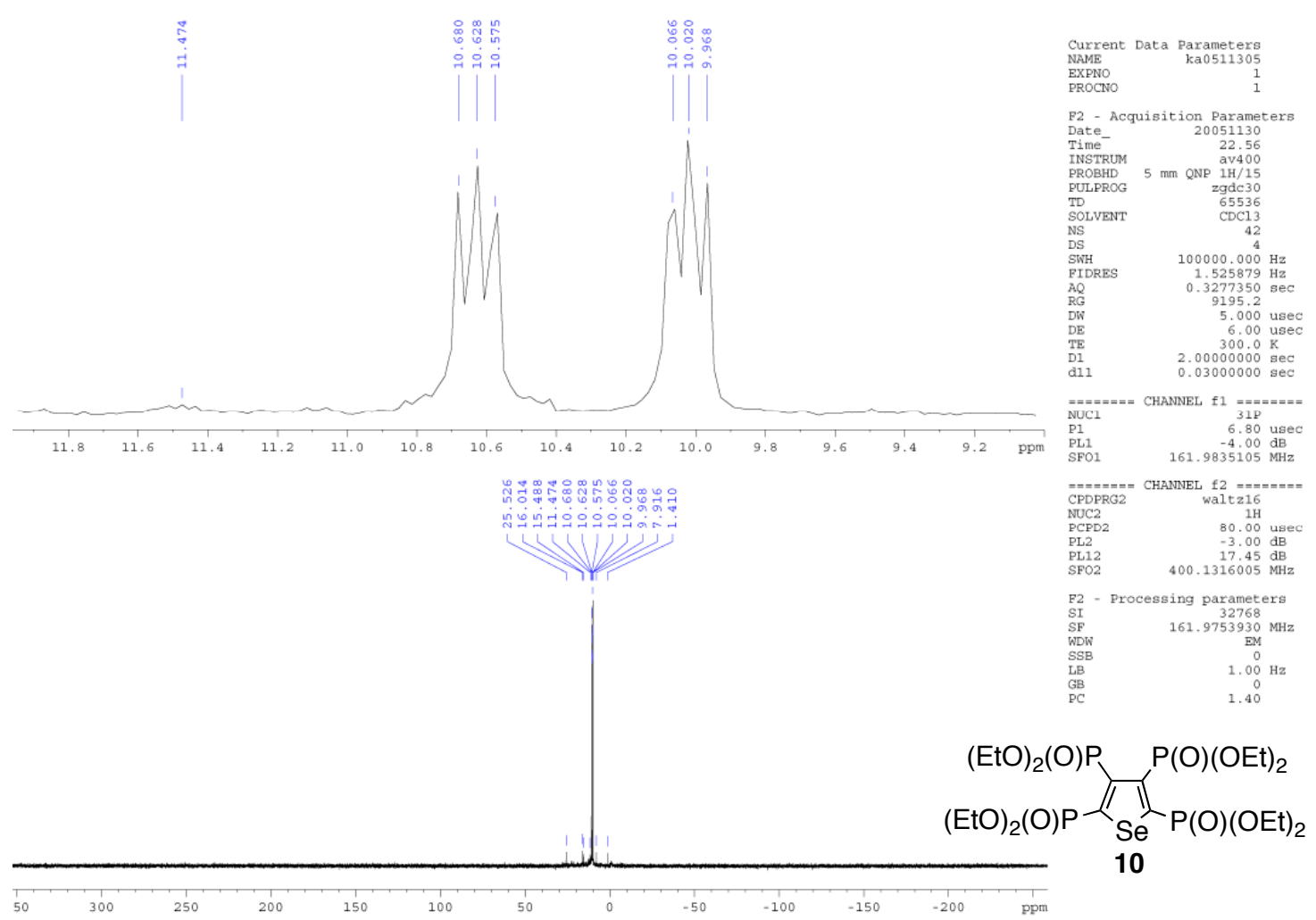

Figure 7-3. ${ }^{31} \mathrm{P}$ NMR (162 MHz, $\left.\mathrm{CDCl}_{3}, 293 \mathrm{~K}\right)$ of $\mathbf{1 0}$ 